\title{
The orbifold-string theories of permutation-type: I. One twisted BRST per cycle per sector
}

\section{M.B. Halpern}

Department of Physics, University of California, Berkeley, CA 94720, U.S.A.

E-mail: halpern@physics.berkeley.edu

ABSTRACT: We resume our discussion of the new orbifold-string theories of permutationtype, focusing in the present series on the algebraic formulation of the general bosonic prototype and especially the target space-times of the theories. In this first paper of the series, we construct one twisted BRST system for each cycle $j$ in each twisted sector $\sigma$ of the general case, verifying in particular the previously-conjectured algebra $\left[\hat{Q}_{i}(\sigma), \hat{Q}_{j}(\sigma)\right]_{+}=0$ of the BRST charges. The BRST systems then imply a set of extended physical-state conditions for the matter of each cycle at cycle central charge $\hat{c}_{j}(\sigma)=26 f_{j}(\sigma)$, where $f_{j}(\sigma)$ is the length of cycle $j$.

KEYwords: Conformal Field Models in String Theory, Bosonic Strings, BRST Symmetry, Discrete and Finite Symmetries

ARXiv EPRINT: 1008.1453 


\section{Contents}

1 Introduction 1

2 BRST in the orbifold program 5

$\begin{array}{lll}3 & \text { Operator-product form of the twisted BRST's } & 8\end{array}$

4 The mode algebras of twisted sector $\sigma \quad 11$

$\begin{array}{lll}5 & \text { One BRST charge per cycle per sector } & 15\end{array}$

6 The physical states of cycle $j$ in sector $\sigma \quad 16$

$\begin{array}{lll}7 & \text { Mode-ordered form of the twisted ghost systems } & 17\end{array}$

8 The extended physical-state conditions $\quad 19$

9 Conclusions $\quad 23$

$\begin{array}{ll}\text { A Fermi statistics for } \hat{c} \text { 's } & 24\end{array}$

B Extended actions and generalized orientation orbifolds 25

\section{Introduction}

The orbifold program [1-15] systematically constructs the twisted sectors of the orbifold conformal field theory $A(H) / H$ from any current-algebraic conformal field theory $A(H)$ with a finite symmetry group $H$. A short review of the program at the conformal-fieldtheoretic level is included in ref. [10].

Opening a new, more phenomenological chapter of the program, I have recently proposed [16-20] that a very simple subset of these orbifolds, called the orbifold-string theories of permutation-type, can provide new physical string theories at multiples of conventional critical central charges.

This class of theories begins by choosing the conformal field theory $A(H)$ to be a set of copies of any critical closed string (hence only Abelian currents), and there are many of 
these, including the bosonic prototypes [16]

$$
\begin{aligned}
\frac{\mathrm{U}(1)^{26 K}}{H_{+}} & =\frac{\mathrm{U}(1)_{1}^{26} \times \ldots \times \mathrm{U}(1)_{K}^{26}}{H_{+}}, & & H_{+} \subset H(\text { perm })_{K} \times H_{26}^{\prime} \\
\frac{\mathrm{U}(1)^{26}}{H_{-}} & =\frac{\mathrm{U}(1)_{L}^{26} \times \mathrm{U}(1)_{R}^{26}}{H_{-}}, & & H_{-} \subset \mathbb{Z}_{2}(\text { w.s. }) \times H_{26}^{\prime} \\
& {\left[\frac{\mathrm{U}(1)^{26 K}}{H_{+}}\right]_{\text {open }} } & &
\end{aligned}
$$

and orbifold-superstring generalizations of these. In this notation, $\mathrm{U}(1)^{26}$ is the critical bosonic closed string at central charge 26 and $\mathrm{U}(1)_{L, R}^{26}$ are the left- and right-movers of $\mathrm{U}(1)^{26}$. The automorphism group $H(\text { perm })_{K}$ is any permutation group on $K$ elements (the copies), the non-trivial element of $\mathbb{Z}_{2}$ (w.s.) is the exchange $L \leftrightarrow R$ of the chiral components, and the automorphism group $H_{26}^{\prime}$ operates uniformly on each copy of $\mathrm{U}(1)^{26}$, including $\mathrm{U}(1)_{L}^{26}$ and $\mathrm{U}(1)_{R}^{26}$. The divisors $H_{ \pm}$can be any subgroup of the direct products shown.

The sets shown in eq. (1.1a) are called the generalized permutation orbifolds $[9,15,16$, 18, 20], with every sector $\sigma$ being a twisted closed string at central charge $\hat{c}(\sigma)=26 K$. The second sets in eq. (1.1b) are the orientation orbifolds $[12,13,15-19]$, with an equal number of twisted open strings at $\hat{c}(\sigma)=52$ and twisted closed strings at $\hat{c}(\sigma)=26$. The closed-string sectors of the orientation orbifolds form the ordinary space-time orbifolds $\mathrm{U}(1)^{26} / H_{26}^{\prime}$, which we will discuss separately elsewhere. The last sets in eq. (1.1c) are the so-called "open-string generalized permutation orbifolds" with all sectors at $\hat{c}(\sigma)=26 \mathrm{~K}$. These sets are constructed along with their branes in refs. $[15,16]$ from the left-movers of the generalized permutation orbifolds. The $\hat{c}=52$ open-string sectors of the orientation orbifolds are included among the $T$-dual families of $\left[\mathrm{U}(1)^{52} / H_{+}\right]_{\text {open }}$, but this is the only case in the third set whose closure to closed strings has been fully studied.

In fact, there exist other bosonic prototypes (see appendix B of this paper), but our discussion in the present series will be limited to the sets shown in eq. (1.1).

A central issue in all these theories is the presence of extra negative-norm states in the conformal field theories of the twisted sectors. (These "ghost" states are formally associated to the time-like direction of each untwisted closed-string copy in the untwisted sector, but these ghosts are removed in the untwisted sector by the physical state conditions and gauges of each copy, leaving $\mathrm{K}$ decoupled copies of the ordinary ghost-free closed string.) We also know that the orbifold program constructs the twisted sectors of any orbifold from the untwisted sector and in string theory we do not expect that orbifoldization would create negative-norm physical states where there are none in the untwisted sectors [21]. This observation implies that the twisted sectors of the new string theories in fact possess new extended world-sheet geometries - including new twisted world-sheet permutation gravities [16], new twisted BRST systems [17], new extended physical-state conditions [17-20] and new gauges at the interacting level [19, 20]. All these phenomena are associated with the existence of the so-called orbifold Virasoro algebras [1, 2, 9, 16-20] (see eqs. (4.3) and (8.1b)), which appear universally in every twisted sector of the orbifolds 
of permutation-type. It is therefore possible, but so far demonstrated only in subexamples $[19,20]$, that the new world-sheet geometries can eliminate all negative-norm states in the orbifold-string theories of permutation-type.

A closely-related issue is the target space-time interpretation of the new string theories, especially since detailed analysis of the physical states of the $\hat{c}(\sigma)=52$ twisted sectors has found an equivalent, reduced formulation [17] of all the $\hat{c}(\sigma)=52$ spectral problems at reduced central charge $c(\sigma)=26$ ! In the reduced formulation, one sees only (a unitary transformation of) the conventional number of negative-norm states subject in fact to the conventional physical-state condition. The reduced formulation is therefore central in providing a strong suggestion that at least some of these theories can describe conventional (single-time) target space-times, with no ghosts in their physical spectra.

As noted above, some simple subexamples of these phenomena have already been studied. Thus, for example, in spite of the half-integral moding of its $\hat{c}=52$ twisted open-string sector, the simplest orientation-orbifold string system with

$$
\mathbb{Z}_{2}(w . s .)=\left(1, \tau_{-}\right), \quad H_{-}=\left(1 \times(1)_{26} ; \tau_{-} \times(-1)_{26}\right)
$$

has been shown to be equivalent [18], even at the interacting level [19], to the ordinary untwisted 26-dimensional open-closed string system. In this sense, "orientation orbifolds include orientifolds" [19] in a rising sequence of ever-more twisted open-closed string systems, with the familiar untwisted critical prototype at the bottom of the hierarchy. Similarly, the pure cyclic permutation-orbifold string systems $H_{+}=\mathbb{Z}_{K}, K=$ prime have been shown [20] to be equivalent at the interacting level to special modular-invariant collections of ordinary closed 26-dimensional strings, while more general $H_{26}^{\prime} \subset H_{+}$corresponds to generically new permutation-orbifold string theories.

In succeeding papers of this series, we will demonstrate by construction that more general choices of $H_{26}^{\prime} \subset H_{ \pm}$can in fact describe a variety of (single-time) target spacetimes in the new theories, including in particular Lorentzian target space-times with sectordependent dimensionality $D(\sigma) \leq 26$ ! In the succeeding papers we will also discuss further evidence for the no-ghost conjecture [16] in the Lorentzian systems, and point out that these theories can sometimes contain a unique graviton (e.g. the orientation-orbifold string theories) or - more often - multiple, presumably-decoupled gravitons (as in the untwisted closed-string sectors for higher $\mathrm{K}$ ).

Following the development of refs. [16-20], our task in this first paper of the new series is to begin the quantization of the full list of theories in eq. (1.1). More precisely, we present here the BRST quantization $[22,23]$ of the generalized permutation orbifoldstring theories (1.1a) and their open-string counterparts (1.1b), all of whose sectors live at matter central charge $\hat{c}(\sigma)=26 K$. (As noted above the twisted open-string sectors of the orientation-orbifold string theories are included in the case $K=2$, and in fact the BRST quantization of each closed-string in $\mathrm{U}(1)^{26} / H_{26}^{\prime}$ is quite ordinary.)

This paper in scope and in detail is therefore a generalization of the BRST quantization of $\hat{c}=52$ matter in ref. [17]. In particular, we follow ref. [17] in obtaining the general BRST quantization directly from the principle of local isomorphisms [3-15], leaving an equivalent 
derivation from the extended actions of the theories (see ref. [16] and appendix B) for another time and place.

The mathematics needed for the generalization is minimal. In orbifold theory, each twisted sector $\sigma$ corresponds to an equivalence class $\sigma$ of the divisor, and an appropriate language here is the familiar $[7,9,16,18]$ cycle-basis for each sector $\sigma$ of the general permutation group $H(\text { perm })_{K}$ :

$$
\hat{\bar{j}}=0,1, \ldots, f_{j}(\sigma)-1, \quad j=0,1, \ldots, N(\sigma)-1, \quad \sum_{j} f_{j}(\sigma)=K .
$$

In this notation, $f_{j}(\sigma)$ and $N(\sigma)$ are respectively the length of cycle $j$ and the number of cycles in sector $\sigma$, while $\overline{\hat{j}}$ indexes within each cycle. For the convenience of the reader, the cycle-data for the elements of $\mathbb{Z}_{K}$ and $S_{K}$ are included in the text. At any stage of the development, the results of ref. [17] can be obtained from the cycle data

$$
K=2, \quad N(1)=1, \quad \hat{\bar{j}} \rightarrow \bar{u}=0, \quad 1, \quad f_{0}(1)=2
$$

for the single non-trivial element $(\sigma=1)$ of $H$ (perm $)_{2}=\mathbb{Z}_{2}$ or $\mathbb{Z}_{2}$ (w.s.).

The results presented here include the twisted ghost and BRST systems of each cycle $j$ of each twisted sector $\sigma$ of all these orbifold-string systems, including in particular the extended BRST algebra of sector $\sigma$

$$
\left[\hat{Q}_{j}(\sigma), \hat{Q}_{l}(\sigma)\right]_{+}=0, \forall j, l \text { in sector } \sigma
$$

which was conjectured in ref. [17]. In a convention we follow throughout this paper, these are the BRST charges of the open-string analogues (1.1c) only, and right-mover copies of each result must be added to describe the generalized permutation orbifolds (1.1a).

The BRST systems then imply the extended physical-state conditions on the matter of cycle $j$ in sector $\sigma$

$$
\begin{aligned}
\left(\hat{L}_{\hat{j} j}\left(\left(m+\frac{\hat{j}}{f_{j}(\sigma)}\right) \geq 0\right)-\hat{a}_{f_{j}(\sigma)} \delta_{m+\frac{\hat{j}}{f_{j}(\sigma)}, 0}\right)|\chi(\sigma)\rangle_{j} & =0 \\
\hat{c}_{j}(\sigma)=26 f_{j}(\sigma), \quad \hat{a}_{f_{j}(\sigma)} & =\frac{13 f_{j}^{2}(\sigma)-1}{12 f_{j}(\sigma)}
\end{aligned}
$$

where $\left\{\hat{L}_{\hat{j} j}\right\}$ are the orbifold Virasoro generators (see eq. (4.3a)) with cycle central charge $\hat{c}_{j}(\sigma)$ and the quantity $\hat{a}_{f_{j}(\sigma)}$ is called the intercept of cycle $j$ in sector $\sigma$. These results generalize those of ref. [17] ( $\hat{c}=52$ and $\left.\hat{a}_{2}=17 / 8\right)$, and special cases of eq. (1.6) were also obtained at the interacting level for $H_{+}=H(\text { perm) })_{K}=\mathbb{Z}_{K}, K=$ prime (where the $K-1$ twisted sectors are single cycles with $\left.f_{0}(\sigma)=K\right)$. The expected sector central charges of the matter

$$
\hat{c}(\sigma)=\sum_{j} \hat{c}_{j}(\sigma)=26 K
$$

are obtained from eqs. (1.3) and (1.6b) by summing over the cycles of each sector. The extended physical-state conditions in eq. (1.6) are the quantum analogues of the classical 
extended Virasoro constraints $\left\{\hat{L}_{\hat{j} j}=0\right\}$ obtained from the extended actions of these theories in ref. [16].

Because they descend universally from the twisted permutation gravities of ref. [16], the BRST systems presented here depend only on the permutation groups $H$ (perm $)_{K}$ or $\mathbb{Z}_{2}$ (w.s.). The automorphism groups $H_{26}^{\prime}$ in eq. (1.1) are however encoded in the explicit forms of the orbifold Virasoro generators $\left\{\hat{L}_{\hat{j} j}\right\}$ of the matter, which will be constructed in the next paper of this series. There we will also discuss the equivalent, reduced formulation of the physical states of each cycle at reduced cycle central charge $c_{j}(\sigma)=26$, and begin our study of the target space-times of the new string theories.

\section{BRST in the orbifold program}

We sketch here the standard steps of the orbifold program [3-15], which systematically constructs the operator-products of the twisted sectors of any orbifold from the operatorproducts of the untwisted sector.

In this case we are interested in obtaining the BRST systems of the generalized permutation orbifolds and their open-string analogues

$$
\frac{\mathrm{U}(1)^{26 K}}{H_{+}}, \quad\left[\frac{\mathrm{U}(1)^{26 K}}{H_{+}}\right]_{\text {open }}, \quad H_{+} \subset H(\text { perm })_{K} \times H_{26}^{\prime}
$$

so we begin with $K$ copies of the operator-product form of the untwisted BRST system given for $K=2$ in ref. [17]. This includes the composite operators

$$
\begin{aligned}
J_{I}(z) & =: \bar{\psi}_{I}(z) \psi_{I}(z):, \quad I=0, \ldots, K-1 \\
T_{I}^{G}(z) & =-\frac{1}{2}: \bar{\psi}_{I}(z) \stackrel{\leftrightarrow}{\partial} \psi_{I}(z):+\frac{3}{2} \partial J_{I}(z) \\
& =: \bar{\psi}_{I}(z) \partial \psi_{I}(z):+2 \partial \bar{\psi}_{I}(z) \psi_{I}(z) \\
J_{I}^{B}(z) & =\bar{\psi}_{I}(z) T_{I}(z)+\frac{1}{2}: \bar{\psi}_{I}(z) T_{I}^{G}(z): \\
T_{I}^{t}(z) & =T_{I}(z)+T_{I}^{G}(z)
\end{aligned}
$$

as well as their operator products, e.g.,

$$
\begin{aligned}
\psi_{I}(z) \bar{\psi}_{J}(w)= & \frac{\delta_{I J}}{(z-w)}+: \psi_{I}(z) \bar{\psi}_{J}(w): \\
T_{I}^{G}(z) T_{J}^{G}(w)= & \delta_{I J}\left\{\frac{-(26 / 2)}{(z-w)^{4}}+\left(\frac{2}{(z-w)^{2}}+\frac{1}{z-w} \partial_{w}\right) T_{I}^{G}(w)\right\} \\
& +T_{I}^{G}(z) T_{J}^{G}(w) \\
T_{I}(z) T_{J}(w)= & \delta_{I J}\left\{\frac{(26 / 2)}{(z-w)^{4}}+\left(\frac{2}{(z-w)^{2}}+\frac{1}{z-w} \partial_{w}\right) T_{I}(w)\right\} \\
& +: T_{I}(z) T_{J}(z):
\end{aligned}
$$

Here $\{\psi, \bar{\psi}\}$ are $\mathrm{K}$ copies of the ghosts, taken as half-integer moded Bardakci-Halpern fermions [24], while $\{J\},\left\{T^{G}\right\}$ and $\left\{J^{B}\right\}$ are the corresponding copies of the ghost current, 
the $c=-26$ ghost stress-tensor and the BRST current respectively. The independent quantities $\{T\}$ are the copies of the $c=26$ matter stress tensor while $\left\{T^{t}\right\}$ are the total stress-tensor copies, each with $c=0$. Finally, as seen in eq. (2.3), the symbol : ...: is operator-product normal ordering.

We remind the reader that here, and throughout the paper the steps are shown as above only for a single set of local operators, which is adequate to describe the openstring analogues in eq. (1.1c). A second set of right-mover operators is required to describe the closed strings of the generalized permutation orbifolds (1.1a). To obtain the twisted sectors of the orbifolds we will apply the principle of local automorphisms [3-15] (including monodromies), but for the open-string analogues this is only a simple shortcut known [15$18]$ to give the correctly-twisted open-string mode algebras. ${ }^{1}$

The automorphic response of all these fields $\{A\}$ to an arbitrary element of the general permutation group of order $K$ is

$$
A_{I}(z)^{\prime}=\omega\left(h_{\sigma}\right)_{I}^{J} A_{J}(z), \quad \omega\left(h_{\sigma}\right) \in H(\text { perm })_{K} .
$$

The next step in the orbifold program is the construction for each operator $A$ the corresponding eigenfield $\mathcal{A}$, whose automorphic response $\mathcal{A}^{\prime}$ to each $\omega\left(h_{\sigma}\right)$ is diagonal:

$$
\begin{aligned}
\mathcal{A}_{\hat{j} j}(z, \sigma) & \equiv \chi_{\hat{j} j}(\sigma) \mathrm{U}(\sigma)_{\hat{j} j}{ }^{I} A_{I}(z, \sigma) \\
\mathcal{A}_{\hat{j} j}(z, \sigma)^{\prime} & =e^{-2 \pi i \frac{\hat{j}}{f_{j}(\sigma)}} \mathcal{A}_{\hat{j} j}(z, \sigma) \\
\mathcal{A}_{\hat{j} \pm f_{j}(\sigma), j}(z, \sigma) & =\mathcal{A}_{\hat{j} j}(z, \sigma) \\
\overline{\hat{j}} & =0, \ldots, f_{j}(\sigma)-1, \quad j=0, \ldots, N(\sigma)-1 \\
\sum_{j} & =N(\sigma), \quad \sum_{j} f_{j}(\sigma)=K .
\end{aligned}
$$

Here $\mathrm{U}(\sigma)$ is the unitary eigenmatrix of the eigenvalue problem of each element $\omega\left(h_{\sigma}\right) \in$ $H(\text { perm })_{K}$, which is known $[7,9,16,18]$ in the cycle-basis of each element: $N(\sigma)$ is the total number of cycles $j$ in each $\omega\left(h_{\sigma}\right)$, while $\hat{j}$ indexes within each cycle $j$ of length $f_{j}(\sigma) \geq 1$. I have also included the standard periodicity convention $(2.5 \mathrm{c})$ within each cycle (which the eigenfields inherit from the eigenvalue problems) and $\overline{\hat{j}}$ is the pullback of $\hat{j}$ to its fundamental region.

In orbifold theory, each sector $\sigma$ corresponds to an equivalence class $\sigma$ of the automorphism group, so we need to choose one representative $\omega\left(h_{\sigma}\right)$ of each equivalence class of $H(\text { perm })_{K}$. For example, each ordered partition of $K$

$$
\sum_{j} f_{j}(\sigma)=K, \quad 1 \leq f_{j+1}(\sigma) \leq f_{j}(\sigma)
$$

\footnotetext{
${ }^{1}$ In fact of course twisted open strings generally have no monodromies, their fractional modeing being associated instead with the presence of different branes at each end of the string. See in particular ref. [15] where the general twisted open WZW string and its branes are constructed from the left-movers of the general WZW orbifold.
} 
defines an equivalence class of the symmetric group $H(\text { perm })_{K}=S_{K}$. The cyclic group $H(\text { perm })_{K}=\mathbb{Z}_{K}$ has $K$ sectors

$$
\omega\left(h_{\sigma}\right)=e^{2 \pi i \frac{\sigma}{K}}, \quad \sigma=0,1, \ldots, K-1, \quad N(\sigma)=\frac{K}{f_{j}(\sigma)}
$$

where $f_{j}(\sigma)$ is any divisor of $K$. In the cyclic groups $f_{j}(\sigma)$ is also the order of each $\omega\left(h_{\sigma}\right)$. The results of ref. [16] can therefore be obtained from the results of this paper by choosing the non-trivial element $\sigma=1$ of $H$ (perm $)_{2}=\mathbb{Z}_{2}$ with the single cycle-length $f_{0}(1)=2$ (and $\overline{\hat{j}} \rightarrow \bar{u}=0,1$ ). More generally, the untwisted sector $\sigma=0$ is described by the unit element

$$
\overline{\hat{j}}=0, \quad f_{j}(0)=1, \quad N(0)=K
$$

for all $H(\text { perm })_{K}$.

The quantities $\{\chi\}$ in the eigenfields (2.5a) are normalizations, which I choose here as

$$
\chi_{\hat{j} j}(\sigma)= \begin{cases}1 & \text { for ghosts } \\ f_{j}(\sigma) & \text { for BRST currents } \\ \sqrt{f_{j}(\sigma)} & \text { otherwise. }\end{cases}
$$

The last (square-root) convention here is the standard choice for permutation-orbifold matter $[7,9,16-18]$ in the orbifold program, and the other conventions for the fermionic operators are consistent with the choice in ref. [17] for the non-trivial element of $H$ (perm) $)_{2}=\mathbb{Z}_{2}$ with $f_{j}(\sigma)=2$. It is then straightforward to compute the operator-products of the eigenfields in terms of the eigenfields themselves (see the remark after eq. (3.5)).

The final step in the orbifold program is an application of the principle of local isomorphisms [3-18], which maps the eigenfields $\{\mathcal{A}\}$ of sector $\sigma$ to the twisted fields $\{\hat{A}\}$ of sector $\sigma$ :

$$
\mathcal{A}_{\hat{j} j}(z, \sigma) \rightarrow \hat{A}_{\hat{j} j}(z, \sigma) .
$$

There are two components to this principle. First, the twisted fields are locally isomorphic to the eigenfields, that is, they have the same operator products. Second, the monodromies of each twisted field $\hat{A}$

$$
\hat{A}_{\hat{j} j}\left(z e^{2 \pi i}, \sigma\right)=e^{-2 \pi i \frac{\hat{j}}{f_{j}(\sigma)}} \hat{A}_{\hat{j} j}(z, \sigma)
$$

is the same as the diagonal automorphic response (2.5b) of the corresponding eigenfield $\mathcal{A}$. In this last step, and only here, do we change from the untwisted Hilbert space to the twisted Hilbert space of sector $\sigma$. The final result for the operator-product form of the twisted BRST system is presented in the following section.

For perspective, we also mention an equivalent, alternate route (via the principle of local isomorphisms and a monodromy decomposition) to the same twisted fields $\hat{A}$. This route and the one described above via eigenfields are both summarized in the so-called commuting diagrams $[3,5,11]$ of the orbifold program. 


\section{Operator-product form of the twisted BRST's}

The twisted fields of cycle $j$ in sector $\sigma$ are as follows

$$
\begin{aligned}
\hat{J}_{\hat{j} j}(z, \sigma) & =\sum_{\hat{l}=0}^{f_{j}(\sigma)-1}: \hat{\bar{\psi}}_{\hat{l} j}(z, \sigma) \psi_{\hat{j}-\hat{l}, j}(z, \sigma): \\
\hat{\theta}_{\hat{j} j}^{G}(z, \sigma) & =-\frac{1}{2} \sum_{\hat{l}=0}^{f_{j}(\sigma)-1}: \hat{\bar{\psi}}_{\hat{l} j}(z, \sigma) \overleftrightarrow{\partial} \hat{\psi}_{\hat{j}-\hat{l}, j}(z, \sigma):+\frac{3}{2} \partial \hat{J}_{\hat{j} j}(z, \sigma) \\
& =\sum_{\hat{l}=0}^{f_{j}(\sigma)-1}:\left(\hat{\bar{\psi}}_{\hat{l} j}(z, \sigma) \partial \hat{\psi}_{\hat{j}-\hat{l}, j}(z, \sigma)+2 \partial \hat{\bar{\psi}}_{\hat{l} j}(z, \sigma) \hat{\psi}_{\hat{j}-\hat{l}, j}(z, \sigma)\right): \\
\hat{J}_{\hat{j} j}^{B}(z, \sigma) & =\sum_{\hat{\theta}=0}^{f_{j}(\sigma)-1}\left\{\hat{\bar{\psi}}_{\hat{l} j}(z, \sigma) \hat{\theta}_{\hat{j}-\hat{l}, j}(\sigma)+\frac{1}{2}: \hat{\bar{\psi}}_{\hat{l} j}(z, \sigma) \hat{\theta}_{\hat{j}-\hat{l}, j}^{G}(z, \sigma):\right\} \\
\hat{\theta}_{\hat{j} j}^{t}(z, \sigma) & =\hat{\theta}_{\hat{j} j}(z, \sigma)+\hat{\theta}_{\hat{j} j}^{G}(z, \sigma) \\
\hat{A}_{\hat{j} j}\left(z e^{2 \pi i}, \sigma\right) & =e^{-2 \pi i \frac{\hat{j}}{f_{j}(\sigma)} \hat{A}_{\hat{j} j}(z, \sigma)} \\
\hat{A}_{\hat{j} \pm f_{j}(\sigma), j}(z, \sigma) & =\hat{A}_{\hat{j} j}(z, \sigma) \\
\overline{\hat{j}} & =0, \ldots, f_{j}(\sigma)-1, \quad j=0, \ldots, N(\sigma)-1, \quad \sum_{j} f_{j}(\sigma)=K
\end{aligned}
$$

where $: \ldots$ : is still operator-product normal ordering and I have included the monodromies and periodicities of each twisted field in eqs. (3.1e) and (3.1f) respectively The fields in this list include the twisted reparametrization ghosts $\{\hat{\psi}, \hat{\bar{\psi}}\}$, the twisted ghost currents $\{\hat{J}\}$, the extended ghost stress-tensors $\left\{\hat{\theta}^{G}\right\}$ and the twisted BRST currents $\left\{\hat{J}^{B}\right\}$. Finally $\{\hat{\theta}\}$ and $\left\{\hat{\theta}^{t}\right\}$ are respectively the extended matter stress-tensors and the extended total stress-tensors of cycle $j$ in sector $\sigma$.

The operator products of the twisted fields are also obtained in detail as follows. We begin with the basic operator products of the twisted ghosts and their currents

$$
\begin{aligned}
& \hat{\bar{\psi}}_{\hat{j} j}(z, \sigma) \hat{\psi}_{\hat{l} l}(w, \sigma)=\frac{\delta_{j l} \delta_{\hat{j}+\hat{l}, 0 \bmod f_{j}(\sigma)}}{z-w}+: \hat{\bar{\psi}}_{\hat{j} j}(z, \sigma) \hat{\psi}_{\hat{l} l}(w, \sigma): \\
& \hat{\bar{\psi}}_{\hat{j} j}(z, \sigma) \hat{\bar{\psi}}_{\hat{l l}}(w, \sigma)=: \hat{\bar{\psi}}_{\hat{j} j}(z, \sigma) \hat{\bar{\psi}}_{\hat{l} l}(w, \sigma): \\
& \hat{\psi}_{\hat{j} j}(z, \sigma) \hat{\psi}_{\hat{l l}}(w, \sigma)=: \hat{\psi}_{\hat{j} j}(z, \sigma) \hat{\psi}_{\hat{l l}}(w, \sigma): \\
& \hat{J}_{\hat{j} j}(z, \sigma) \hat{\bar{\psi}}_{\hat{l} l}(w, \sigma)=\frac{\delta_{j l} \hat{\bar{\psi}}_{\hat{j}+\hat{l}, j}(w)}{z-w}+: \hat{J}_{\hat{j} j}(z, \sigma) \hat{\bar{\psi}}_{\hat{l l}}(w, \sigma): \\
& \hat{J}_{\hat{j} j}(z, \sigma) \hat{\psi}_{\hat{l l}}(w, \sigma)=\frac{-\delta_{j l} \hat{\psi}_{\hat{j}+\hat{l}, j}(w)}{z-w}+: \hat{J}_{\hat{j} j}(z, \sigma) \hat{\psi}_{\hat{l} l}(w, \sigma): \\
& \hat{J}_{\hat{j} j}(z, \sigma) \hat{J}_{\hat{l} l}(w, \sigma)=\frac{\delta_{j l} \delta_{\hat{j}+\hat{l}, 0 \bmod f_{j}(\sigma)}}{(z-w)^{2}}+: \hat{J}_{\hat{j} j}(z, \sigma) \hat{J}_{\hat{l} l}(w, \sigma):
\end{aligned}
$$


and continue with the operator products involving the extended stress tensors:

$$
\begin{aligned}
& \hat{\theta}_{\hat{j} j}(z, \sigma) \hat{\theta}_{\hat{l} l}(w, \sigma)=\delta_{j l}\left\{\frac{\left(\frac{26}{2}\right) f_{j}(\sigma) \delta_{\hat{j}+\hat{l}, 0 \bmod f_{j}(\sigma)}}{(z-w)^{4}}\right. \\
& \left.+\left(\frac{2}{(z-w)^{2}}+\frac{1}{z-w} \partial_{w}\right) \hat{\theta}_{\hat{j}+\hat{l}, j}(w, \sigma)\right\} \\
& +: \hat{\theta}_{\hat{j} j}(z, \sigma) \hat{\theta}_{\hat{l} l}(w, \sigma): \\
& \hat{\theta}_{\hat{j} j}^{G}(z, \sigma) \hat{\theta}_{\hat{l} l}^{G}(w, \sigma)=\delta_{j l}\left\{\frac{-\left(\frac{26}{2}\right) f_{j}(\sigma) \delta_{\hat{j}+\hat{l}, 0 \bmod f_{j}(\sigma)}}{(z-w)^{4}}\right. \\
& \left.+\left(\frac{2}{(z-w)^{2}}+\frac{1}{z-w} \partial_{w}\right) \hat{\theta}_{\hat{j}+\hat{l}, j}^{G}(w, \sigma)\right\} \\
& +: \hat{\theta}_{\hat{j} j}^{G}(z, \sigma) \hat{\theta}_{\hat{l l}}^{G}(w, \sigma): \\
& \hat{\theta}_{\hat{j} j}^{t}(z, \sigma) \hat{\theta}_{\hat{l} l}^{t}(w, \sigma)=\delta_{j l}\left(\frac{2}{(z-w)^{2}}+\frac{1}{z-w} \partial_{w}\right) \hat{\theta}_{\hat{j}+\hat{l}, j}^{t}(w, \sigma) \\
& +: \hat{\theta}_{j j}^{t}(z, \sigma) \hat{\theta}_{\hat{l l}}^{t}(w, \sigma) \text { : } \\
& \hat{\theta}_{\hat{j} j}^{G}(z, \sigma) \hat{\bar{\psi}}_{\hat{l l}}(w, \sigma)=\delta_{j l}\left(-\frac{1}{(z-w)^{2}}+\frac{1}{z-w} \partial_{w}\right) \bar{\psi}_{\hat{j}+\hat{l}, j}(w, \sigma) \\
& +: \hat{\theta}_{\hat{j} j}^{G}(z, \sigma) \hat{\bar{\psi}}_{\hat{l l}}(w, \sigma): \\
& \hat{\theta}_{\hat{j} j}^{G}(z, \sigma) \hat{\psi}_{\hat{l} l}(w, \sigma)=\delta_{j l}\left(\frac{2}{(z-w)^{2}}+\frac{1}{z-w} \partial_{w}\right) \hat{\psi}_{\hat{j}+\hat{l}, j}(w, \sigma) \\
& +: \hat{\theta}_{\hat{j} j}^{G}(z, \sigma) \hat{\psi}_{\hat{l l}}(w, \sigma): \\
& \hat{\theta}_{\hat{j} j}^{G}(z, \sigma) \hat{J}_{\hat{l l}}(w, \sigma)=\delta_{j l}\left(\frac{1}{(z-w)^{2}}+\frac{1}{z-w} \partial_{w}\right) \hat{J}_{\hat{j}+\hat{l}, j}(w) \\
& +: \hat{\theta}_{\hat{j} j}^{G}(z, \sigma) \hat{J}_{\hat{l} l}(w, \sigma): .
\end{aligned}
$$

Finally, we give the operator products which involve the twisted BRST currents:

$$
\begin{aligned}
\hat{J}_{\hat{j} j}^{B}(z, \sigma) \hat{\psi}_{\hat{l} l}(w, \sigma)= & \delta_{j l}\left(\frac{\hat{J}_{\hat{j}+\hat{l}, j}(w, \sigma)}{(z-w)^{2}}+\frac{\hat{\theta}_{\hat{j}+\hat{l}, j}^{t}(w, \sigma)}{z-w}\right) \\
& +: \hat{J}_{\hat{j} j}^{B}(z, \sigma) \hat{\psi}_{\hat{l} l}(w, \sigma): \\
\hat{\theta}_{\hat{j} j}^{t}(z, \sigma) \hat{J}_{\hat{l} l}^{B}(w, \sigma)= & \delta_{j l}\left(\frac{1}{(z-w)^{2}}+\frac{1}{z-w} \partial_{w}\right) \hat{J}_{\hat{j}+\hat{l}, j}^{B}(w) \\
& +: \hat{\theta}_{\hat{j} j}^{t}(z, \sigma) \hat{J}_{\hat{l} l}^{B}(w, \sigma):
\end{aligned}
$$




$$
\begin{aligned}
& \hat{J}_{\hat{j} j}^{B}(z, \sigma) \hat{J}_{\hat{l} l}^{B}(w, \sigma)= \delta_{j l} f_{j}(\sigma) \sum_{\hat{m}=0}^{f_{j}(\sigma)-1}\left\{\frac{10}{(z-w)^{3}} \partial_{w} \hat{\bar{\psi}}_{\hat{m} j}(w, \sigma) \hat{\bar{\psi}}_{\hat{j}+\hat{l}-\hat{m}, j}(w, \sigma)\right. \\
&+\frac{5}{(z-w)^{2}} \partial_{w}^{2} \bar{\psi}_{\hat{m} j}(w, \sigma) \hat{\bar{\psi}}_{\hat{j}+\hat{l}-\hat{m}, j}(w, \sigma) \\
&\left.+\frac{3}{2(z-w)} \partial_{w}\left(\partial_{w}^{2} \hat{\bar{\psi}}_{\hat{m} j}(w, \sigma) \hat{\bar{\psi}}_{\hat{j}+\hat{l}-\hat{m}, j}(w, \sigma)\right)\right\} \\
&+: \hat{J}_{\hat{j} j}^{B}(z, \sigma) \hat{J}_{\hat{l} l}^{B}(w, \sigma): \\
& \overline{\hat{j}}=0, \ldots, f_{j}(\sigma)-1, \quad j=0, \ldots, N(\sigma)-1, \quad \sum_{j} f_{j}(\sigma)=K .
\end{aligned}
$$

We should also mention that the extended matter stress tensors are independent of the ghost systems

$$
\hat{\theta}_{\hat{j} j}(z, \sigma) \hat{G}_{\hat{l l}}(w, \sigma)=: \hat{\theta}_{\hat{j} j}(z, \sigma) \hat{G}_{\hat{l l}}(w, \sigma):
$$

where $\hat{G}$ can be $\hat{\psi}, \hat{\bar{\psi}}$ or any composite thereof.

Eqs. (3.1) thru (3.4) are a complete description at the operator-product level of the twisted BRST systems of twisted sector $\sigma$. The composite forms and operator products of the (intermediate) eigenfields $\{\mathcal{A}\}$ can in fact be read from these equations by replacing $\hat{A} \rightarrow \mathcal{A}$ and the monodromies (3.1e) by the automorphic responses (2.5b). Note also that the dynamics of twisted sector $\sigma$ is semisimple with respect to the cycles, as seen in the earlier conformal-field-theoretic study of the permutation orbifolds [9].

We close this section with some remarks about the operator-product algebra of the extended stress tensors and their associated ordinary Virasoro subalgebras. From eqs. (3.3a)(3.3c), one reads the central charges of the extended stress-tensors of cycle $j$ in sector $\sigma$

$$
\begin{array}{ll}
\hat{c}_{j}(\sigma)=26 f_{j}(\sigma) & \text { for }\{\hat{\theta}\} \\
\hat{c}_{j}^{G}(\sigma)=-26 f_{j}(\sigma) & \text { for }\left\{\hat{\theta}^{G}\right\} \\
\hat{c}_{j}^{t}(\sigma)=\hat{c}_{j}(\sigma)+\hat{c}_{j}(\sigma)^{G}=0 & \text { for }\left\{\hat{\theta}^{t}\right\} .
\end{array}
$$

In fact these "cycle" central charges are the central charges of the ordinary (Virasoro) stress-tensors $\hat{\theta}_{0 j}, \hat{\theta}_{0 j}^{G}$ and $\hat{\theta}_{0 j}^{t}$ of cycle $j$, whose operator products have the schematic (Virasoro) form

$$
\begin{aligned}
\hat{\theta}_{0 j}(z, \sigma) \hat{\theta}_{0 l}(w, \sigma)= & \delta_{j l}\left(\frac{\left(\hat{c}_{j}(\sigma) / 2\right)}{(z-w)^{4}}+\frac{2}{(z-w)^{2}} \partial_{w}\right) \hat{\theta}_{0 j}(w) \\
& +: \hat{\theta}_{0 j}(z, \sigma) \hat{\theta}_{0 l}(w, \sigma):
\end{aligned}
$$

for each of the three types. More familiar central charges are obtained for the physical stress tensors of sector $\sigma$

$$
\begin{aligned}
\hat{\theta}(z, \sigma) & \equiv \sum_{j} \hat{\theta}_{0 j}(z, \sigma), \quad \hat{\theta}^{G}(z, \sigma) \equiv \sum_{j} \hat{\theta}_{0 j}(z, \sigma) \\
\hat{\theta}^{t}(z, \sigma) & \equiv \hat{\theta}(z, \sigma)+\hat{\theta}^{G}(z, \sigma)
\end{aligned}
$$


all three of which share the schematic (Virasoro) operator product:

$$
\begin{aligned}
\hat{\theta}(z, \sigma) \hat{\theta}(w, \sigma)= & \frac{\hat{c}(\sigma) / 2}{(z-w)^{4}}+\left(\frac{2}{(z-w)^{2}}+\frac{1}{z-w} \partial_{w}\right) \hat{\theta}(w, \sigma) \\
& +: \hat{\theta}(z, \sigma) \hat{\theta}(w, \sigma): \\
\hat{c}(\sigma)= & \sum_{j} \hat{c}_{j}(\sigma)=26 K \\
\hat{c}^{G}(\sigma)= & \sum_{j} \hat{c}_{j}^{G}(\sigma)=-26 K \\
\hat{c}^{t}(\sigma) & =\hat{c}(\sigma)+\hat{c}^{G}(\sigma)=0 .
\end{aligned}
$$

The cycle-sums in $(3.9 \mathrm{~b}),(3.9 \mathrm{c})$ were evaluated with the sum rule in eq. $(3.1 \mathrm{~g})$, and we recognize $26 K$ in eq. $(3.9 \mathrm{~b})$ as the matter central charge of each sector of any permutation orbifold on $K$ copies of $\mathrm{U}(1)^{26}$.

\section{The mode algebras of twisted sector $\sigma$}

Consulting the monodromies (3.1e) and the conformal-weight terms $\left\{\Delta /(z-w)^{2}\right\}$ in the operator products with the extended stress tensors, we define the modes $\left\{\hat{A}\left(m+\frac{\hat{j}}{f_{j}(\sigma)}\right)\right\}$ of each twisted field as follows:

$$
\begin{aligned}
\hat{\bar{\psi}}_{\hat{j} j}(z, \sigma) & =\sum_{m \in \mathbb{Z}} \hat{c}_{\hat{j} j}\left(m+\frac{\hat{j}}{f_{j}(\sigma)}\right) z^{-\left(m+\frac{\hat{j}}{f_{j}(\sigma)}\right)+1} \\
\hat{\psi}_{\hat{j} j}(z, \sigma) & =\sum_{m \in \mathbb{Z}} \hat{b}_{\hat{j} j}\left(m+\frac{\hat{j}}{f_{j}(\sigma)}\right) z^{-\left(m+\frac{\hat{j}}{f_{j}(\sigma)}\right)-2} \\
\hat{J}_{\hat{j} j}(z, \sigma) & =\sum_{m \in \mathbb{Z}} \hat{J}_{\hat{j} j}\left(m+\frac{\hat{j}}{f_{n}(\sigma)}\right) z^{-\left(m+\frac{\hat{j}}{f_{j}(\sigma)}\right)-1} \\
\hat{J}_{\hat{j} j}^{B}(z, \sigma) & =\sum_{m \in \mathbb{Z}} \hat{J}_{\hat{j} j}^{B}\left(m+\frac{\hat{j}}{f_{j}(\sigma)}\right) z^{-\left(m+\frac{\hat{j}}{f_{j}(\sigma)}\right)-1} \\
\hat{\theta}_{\hat{j} j}^{t}(z, \sigma) & =\sum_{m \in \mathbb{Z}} \hat{L}_{\hat{j} j}^{t}\left(m+\frac{\hat{j}}{f_{j}(\sigma)}\right) z^{-\left(m+\frac{\hat{j}}{f_{j}(\sigma)}\right)-2} \\
\hat{L}_{\hat{j} j}^{t}\left(m+\frac{\hat{j}}{f_{j}(\sigma)}\right) & =\hat{L}_{\hat{j} j}\left(m+\frac{\hat{j}}{f_{j}(\sigma)}\right)+\hat{L}_{\hat{j} j}^{G}\left(m+\frac{\hat{j}}{f_{j}(\sigma)}\right) \\
\hat{A}_{\hat{j} \pm f_{j}(\sigma), j}\left(m+\frac{\hat{j} \pm f_{j}(\sigma)}{f_{j}(\sigma)}\right) & =\hat{A}_{\hat{j} j}\left(m \pm 1+\frac{\hat{j}}{f_{j}(\sigma)}\right) .
\end{aligned}
$$

The periodicity $(4.1 \mathrm{~g})$ of the modes is a consequence of the periodicity (3.1f) of the twisted fields.

Then by standard contour methods [5] we find the mode algebra of cycle $j$ in each twisted sector $\sigma$, beginning with the twisted ghosts and their currents

$$
\left[\hat{c}_{\hat{j} j}\left(m+\frac{\hat{j}}{f_{j}(\sigma)}\right), \hat{b}_{\hat{l} l}\left(n+\frac{\hat{l}}{f_{l}(\sigma)}\right)\right]_{+}=\delta_{j l} \delta_{m+n+\frac{\hat{j}+\hat{l}}{f_{j}(\sigma)}, 0}
$$




$$
\begin{aligned}
& {\left[\hat{c}_{\hat{j} j}\left(m+\frac{\hat{j}}{f_{j}(\sigma)}\right), \hat{c}_{\hat{l} l}\left(n+\frac{\hat{l}}{f_{l}(\sigma)}\right)\right]_{+}=\left[\hat{b}_{\hat{j} j}\left(m+\frac{\hat{j}}{f_{j}(\sigma)}\right), \hat{b}_{\hat{l} l}\left(n+\frac{\hat{l}}{f_{l}(\sigma)}\right)\right]_{+}=0} \\
& {\left[\hat{J}_{\hat{j} j}\left(m+\frac{\hat{j}}{f_{j}(\sigma)}\right), \hat{c}_{\hat{l} l}\left(n+\frac{\hat{l}}{f_{l}(\sigma)}\right)\right]=\delta_{j l} \hat{c}_{\hat{j}+\hat{l}, j}\left(m+n+\frac{\hat{j}+\hat{l}}{f_{j}(\sigma)}\right)} \\
& {\left[\hat{J}_{\hat{j} j}\left(m+\frac{\hat{j}}{f_{j}(\sigma)}\right), \hat{b}_{\hat{l} l}\left(n+\frac{\hat{l}}{f_{l}(\sigma)}\right)\right]=-\delta_{j l} \hat{b}_{\hat{j}+\hat{l}, j}\left(m+n+\frac{\hat{j}+\hat{l}}{f_{j}(\sigma)}\right)} \\
& {\left[\hat{J}_{\hat{j} j}\left(m+\frac{\hat{j}}{f_{j}(\sigma)}\right), \hat{J}_{\hat{l} l}\left(m+\frac{\hat{l}}{f_{l}(\sigma)}\right)\right]=\delta_{j l} f_{j}(\sigma)\left(m+\frac{\hat{j}}{f_{j}(\sigma)}\right) \delta_{m+n+\frac{\hat{j}+\hat{l}}{f_{j}(\sigma)}, 0}} \\
& \overline{\hat{j}}=0, \ldots, f_{j}(\sigma)-1, \quad j=0, \ldots, N(\sigma)-1, \quad \sum_{j} f_{j}(\sigma)=K .
\end{aligned}
$$

One consequence of our normalization choice (2.9) is the simple ghost anticommutator (4.2a).

We turn next to the mode algebras of the extended stress tensors, which include the following three extended Virasoro algebras:

$$
\begin{aligned}
& {\left[\hat{L}_{\hat{j} j}\left(m+\frac{\hat{j}}{f_{j}(\sigma)}\right), \hat{L}_{\hat{l} l}\left(n+\frac{\hat{l}}{f_{l}(\sigma)}\right)\right]} \\
& =\delta_{j l}\left\{\left(m-n+\frac{\hat{j}-\hat{l}}{f_{j}(\sigma)}\right) \hat{L}_{\hat{j}+\hat{l}, j}\left(m+n+\frac{\hat{j}+\hat{l}}{f_{j}(\sigma)}\right)\right. \\
& \left.\quad+\frac{26 f_{j}(\sigma)}{12}\left(m+\frac{\hat{j}}{f_{j}(\sigma)}\right)\left(\left(m+\frac{\hat{j}}{f_{j}(\sigma)}\right)^{2}-1\right) \delta_{m+n+\frac{\hat{j}+\hat{l}}{f_{j}(\sigma)}, 0}\right\} \\
& {\left[\begin{array}{c}
\left.\hat{L}_{\hat{j} j}^{G}\left(m+\frac{\hat{j}}{f_{j}(\sigma)}\right), \hat{L}_{\hat{l} l}^{G}\left(n+\frac{\hat{l}}{f_{l}(\sigma)}\right)\right] \\
\quad=\delta_{j l}\left\{\left(m-n+\frac{\hat{j}-\hat{l}}{f_{j}(\sigma)}\right) \hat{L}_{\hat{j}+\hat{l}, j}^{G}\left(m+n+\frac{\hat{j}+\hat{l}}{f_{j}(\sigma)}\right)\right. \\
{\left[\hat{L}_{\hat{j} j}^{t}\left(m+\frac{\hat{j}}{f_{j}(\sigma)}\right), \hat{L}_{\hat{l} l}^{t}\left(n+\frac{\hat{l}}{f_{l}(\sigma)}\right)\right]} \\
\quad=\delta_{j l}\left(m-n+\frac{\left.\hat{j}+\frac{\hat{j}}{f_{j}(\sigma)}\right)}{f_{j}(\sigma)}\right)\left(m+\frac{\hat{l}}{f_{j}(\sigma)}\right)_{\hat{j}+\hat{l}, j}^{t}\left(m+n+\frac{\hat{j}+\hat{l}}{f_{j}(\sigma)}\right) \\
{\left[\hat{L}_{\hat{j} j}\left(m+\frac{\hat{j}}{f_{j}(\sigma)}\right), \hat{L}_{\hat{l} l}^{G}\left(n+\frac{\hat{l}+\hat{l}}{f_{l}(\sigma)}\right)\right]=0 .}
\end{array}\right\}}
\end{aligned}
$$

These algebras are also called general orbifold Virasoro algebras $[1,2,9,16-20]$, and in particular the algebra of the matter generators $\left\{\hat{L}_{\hat{j} j}\right\}$ at fixed $j$ is an orbifold Virasoro 
algebra of order $f_{j}(\sigma)$. Each of the three orbifold Virasoro algebras contains a so-called integral Virasoro subalgebra for each cycle $j$ in sector $\sigma$

$$
\begin{aligned}
\hat{\theta}_{0 j}(z, \sigma) & =\sum_{m \in \mathbb{Z}} \hat{L}_{0 j}(m) z^{-m-2} \\
{\left[\hat{L}_{0 j}(m), \hat{L}_{0 l}(n)\right]=} & \delta_{j l}\left\{(m-n) \hat{L}_{0 j}(m+n)+\frac{\hat{c}_{j}(\sigma)}{12} m\left(m^{2}-1\right) \delta_{m+n, 0}\right\} \\
\hat{c}_{j}(\sigma) & = \begin{cases}26 f_{j}(\sigma) & \text { for }\left\{\hat{L}_{0 j}\right\} \\
-26 f_{j}(\sigma) & \text { for }\left\{\hat{L}_{0 j}^{G}\right\} \\
0 & \text { for }\left\{\hat{L}_{0 j}^{t}\right\}\end{cases}
\end{aligned}
$$

which exhibit the three cycle central charges discussed in the previous section. Because the cycle dynamics is semisimple, there are also three physical Virasoro subalgebras for each sector $\sigma$

$$
\begin{aligned}
\hat{\theta}(z, \sigma) & =\sum_{j} \hat{L}_{\sigma}(m) z^{-m-2} \\
\hat{L}_{\sigma}(m) & \equiv \sum_{j} \hat{L}_{0 j}(m) \\
{\left[\hat{L}_{\sigma}(m), \hat{L}_{\sigma}(n)\right] } & =(m-n) \hat{L}_{\sigma}(m+n)+\frac{\hat{c}(\sigma)}{12} m\left(m^{2}-1\right) \delta_{m+n, 0} \\
\hat{c}(\sigma) & =\sum_{j} \hat{c}_{j}(\sigma)= \begin{cases}26 K & \text { for }\left\{\hat{L}_{\sigma}\right\} \\
-26 K & \text { for }\left\{\hat{L}_{\sigma}^{G}\right\} \\
0 & \text { for }\left\{\hat{L}_{\sigma}^{t}\right\} .\end{cases}
\end{aligned}
$$

which exhibit the expected sector central charges.

Of course, the extended Virasoro generators of the matter are independent of the ghost systems

$$
\left[\hat{L}_{\hat{j} j}\left(m+\frac{\hat{j}}{f_{j}(\sigma)}\right), \hat{G}_{\hat{l} l}\left(n+\frac{\hat{l}}{f_{l}(\sigma)}\right)\right]=0
$$

where $\hat{G}$ can be $\hat{c}, \hat{b}$ or any composite thereof. This statement includes eq. (4.3d) as a special case.

Other algebras in the twisted ghost systems include the following:

$$
\begin{aligned}
& \quad\left[\hat{L}_{\hat{j} j}^{G}\left(m+\frac{\hat{j}}{f_{j}(\sigma)}\right), \hat{c}_{\hat{l} l}\left(n+\frac{\hat{l}}{f_{l}(\sigma)}\right)\right] \\
& \quad=-\delta_{j l}\left(2\left(m+\frac{\hat{j}}{f_{j}(\sigma)}\right)+n+\frac{\hat{l}}{f_{l}(\sigma)}\right) \hat{c}_{\hat{j}+\hat{l}, j}\left(m+n+\frac{\hat{j}+\hat{l}}{f_{j}(\sigma)}\right) \\
& \quad\left[\hat{L}_{\hat{j} j}^{G}\left(m+\frac{\hat{j}}{f_{j}(\sigma)}\right), \hat{b}_{\hat{l} l}\left(n+\frac{\hat{l}}{f_{l}(\sigma)}\right)\right] \\
& \quad=\delta_{j l}\left(m-n+\frac{\hat{j}+\hat{l}}{f_{j}(\sigma)}\right) \hat{b}_{\hat{j}+\hat{l}, j}\left(m+n+\frac{\hat{j}+\hat{l}}{f_{j}(\sigma)}\right)
\end{aligned}
$$




$$
\begin{aligned}
& {\left[\hat{L}_{\hat{j} j}^{G}\left(m+\frac{\hat{j}}{f_{j}(\sigma)}\right), \hat{J}_{\hat{l} l}\left(n+\frac{\hat{l}}{f_{l}(\sigma)}\right)\right]} \\
& \quad=\delta_{j l}\left(m-n+\frac{\hat{j}+\hat{l}}{f_{j}(\sigma)}\right) \hat{J}_{\hat{j}+\hat{l}, j}\left(m+n+\frac{\hat{j}+\hat{l}}{f_{j}(\sigma)}\right) .
\end{aligned}
$$

Finally, we have the algebra which involves the modes of the twisted BRST currents:

$$
\begin{aligned}
& {\left[\hat{L}_{\hat{j} j}^{t}\left(m+\frac{\hat{j}}{f_{j}(\sigma)}\right), \hat{J}_{\hat{l} l}^{B}\left(n+\frac{\hat{l}}{f_{l}(\sigma)}\right)\right]=-\delta_{j l}\left(n+\frac{\hat{l}}{f_{j}(\sigma)}\right) \hat{J}_{\hat{j}+\hat{l}, j}^{B}\left(m+n+\frac{\hat{j}+\hat{l}}{f_{j}(\sigma)}\right)} \\
& {\left[\hat{J}_{\hat{j} j}^{B}\left(m+\frac{\hat{j}}{f_{j}(\sigma)}\right), \hat{b}_{\hat{l} l}\left(n+\frac{\hat{l}}{f_{l}(\sigma)}\right)\right]_{+}} \\
& =\delta_{j l}\left\{\left(m+\frac{\hat{j}}{f_{j}(\sigma)}\right) \hat{J}_{\hat{j}+\hat{l}, j}\left(m+n+\frac{\hat{j}+\hat{l}}{f_{j}(\sigma)}\right)+\hat{L}_{\hat{j}+\hat{l}, j}^{t}\left(m+n+\frac{\hat{j}+\hat{l}}{f_{j}(\sigma)}\right)\right\} \\
& {\left[\hat{J}_{\hat{j} j}^{B}\left(m+\frac{\hat{j}}{f_{j}(\sigma)}\right), \hat{J}_{\hat{l} l}^{B}\left(n+\frac{\hat{l}}{f_{l}(\sigma)}\right)\right]_{+}} \\
& =\delta_{j l} f_{j}(\sigma)\left\{5\left(m+\frac{\hat{j}}{f_{j}(\sigma)}\right)\left(n+\frac{\hat{l}}{f_{j}(\sigma)}\right)-\frac{3}{2}\left(m+n+\frac{\hat{j}+\hat{l}}{f_{j}(\sigma)}\right)\left(m+n+\frac{\hat{j}+\hat{l}}{f_{j}(\sigma)}-1\right)\right\} \\
& \left.\times \sum_{\hat{m}=0}^{f_{j}(\sigma)-1} \sum_{p \in \mathbb{Z}}\left(p+\frac{\hat{m}}{f_{j}(\sigma)}\right) \hat{c}_{\hat{m} j}\left(p+\frac{\hat{m}}{f_{j}(\sigma)}\right]\right) \hat{c}_{\hat{j}+\hat{l}-\hat{m}, j}\left(m+n-p+\frac{\hat{j}+\hat{l}-\hat{m}}{f_{j}(\sigma)}\right) \\
& \overline{\hat{j}}=0, \ldots, f_{j}(\sigma)-1, \quad j=0, \ldots, N(\sigma)-1, \quad \sum_{j} f_{j}(\sigma)=K .
\end{aligned}
$$

Appendix A includes a technical remark used to simplify the right side of the anticommutator (4.8c) of the BRST current modes. Also, evaluation of this anticommutator at $f_{j}(\sigma)=2$ shows a (non-propagating) typo in eq. (4.4i) of ref. [17], where the last factor before the sums should read $\left(m+n+\frac{u+v}{2}-1\right)$ instead of $\left(m+n+\frac{u+v}{2}+1\right)$.

We conclude with the operator-product normal-ordered forms of the composite mode operators themselves

$$
\begin{aligned}
& \hat{J}_{\hat{j} j}\left(m+\frac{\hat{j}}{f_{j}(\sigma)}\right)=\sum_{\hat{l}=0}^{f_{j}(\sigma)-1} \sum_{p \in \mathbb{Z}}: \hat{c}_{\hat{l} j}\left(p+\frac{\hat{l}}{f_{j}(\sigma)}\right) \hat{b}_{\hat{j}-\hat{l}, j}\left(m-p+\frac{\hat{j}-\hat{l}}{f_{j}(\sigma)}\right): \\
& \hat{L}_{\hat{j} j}^{G}\left(m+\frac{\hat{j}}{f_{j}(\sigma)}\right) \\
& =-\sum_{\hat{l}=0}^{f_{j}(\sigma)-1} \sum_{p \in \mathbb{Z}}\left(m+p+\frac{\hat{j}+\hat{l}}{f_{j}(\sigma)}\right): \hat{c}_{\hat{l} j}\left(p+\frac{\hat{l}}{f_{j}(\sigma)}\right) \hat{b}_{\hat{j}+\hat{l}, j}\left(m-p+\frac{\hat{j}-\hat{l}}{f_{j}(\sigma)}\right):
\end{aligned}
$$




$$
\begin{aligned}
& \hat{J}_{\hat{j} j}^{B}\left(m+\frac{\hat{j}}{f_{j}(\sigma)}\right) \\
&=\sum_{\hat{l}=0}^{f_{j}(\sigma)-1} \sum_{p \in \mathbb{Z}}\left\{\hat{c}_{\hat{l} j}\left(p+\frac{\hat{l}}{f_{j}(\sigma)}\right) \hat{L}_{\hat{j}-\hat{l}, j}\left(m-p+\frac{\hat{j}-\hat{l}}{f_{j}(\sigma)}\right)+\right. \\
&\left.+\frac{1}{2}: \hat{c}_{\hat{l} j}\left(p+\frac{\hat{l}}{f_{j}(\sigma)}\right) \hat{L}_{\hat{j}-\hat{l}, j}^{G}\left(m-p+\frac{\hat{j}-\hat{l}}{f_{j}(\sigma)}\right):\right\}
\end{aligned}
$$

but these forms are not as useful as the mode-ordered forms of these operators given in section 7 .

\section{One BRST charge per cycle per sector}

In the discussion above, we have constructed the modes $\left\{\hat{J}_{\hat{j} j}^{B}\left(m+\hat{j} / f_{j}(\sigma)\right)\right\}$ of one twisted BRST current per cycle per sector for the general bosonic orbifold-string system of permutation-type. We may then define exactly one BRST charge per cycle per sector

$$
\hat{Q}_{j}(\sigma) \equiv \hat{J}_{0 j}^{B}(0), \quad j=0,1, \ldots, N(\sigma)-1
$$

as the unique zero mode $(m=\hat{j}=0)$ of each twisted BRST current.

As a consequence, the results above imply the following algebra of the BRST charges with the other operators in sector $\sigma$

$$
\begin{aligned}
& {\left[\hat{Q}_{j}(\sigma), \hat{L}_{\hat{l} l}^{t}\left(n+\frac{\hat{l}}{f_{l}(\sigma)}\right)\right]=0, \forall j, l, \sigma} \\
& {\left[\hat{Q}_{j}(\sigma), \hat{b}_{\hat{l} l}\left(n+\frac{\hat{l}}{f_{l}(\sigma)}\right)\right]_{+}=\delta_{j l} \hat{L}_{\hat{l} j}^{t}\left(n+\frac{\hat{l}}{f_{j}(\sigma)}\right)} \\
& {\left[\hat{Q}_{j}(\sigma), \hat{J}_{\hat{l} l}^{B}\left(n+\frac{\hat{l}}{f_{l}(\sigma)}\right)\right]_{+}} \\
& =-\delta_{j l} \frac{3}{2} f_{j}(\sigma)\left(n+\frac{\hat{l}}{f_{j}(\sigma)}\right)\left(n+\frac{\hat{l}}{f_{j}(\sigma)}-1\right) \\
& \quad \times \sum_{\hat{j}=0}^{f_{j}(\sigma)-1} \sum_{p \in \mathbb{Z}}\left(p+\frac{\hat{j}}{f_{j}(\sigma)}\right) \hat{c}_{\hat{j} j}\left(p+\frac{\hat{j}}{f_{j}(\sigma)}\right) \hat{c}_{\hat{l}-\hat{j}, j}\left(n-p+\frac{\hat{l}-\hat{j}}{f_{j}(\sigma)}\right)
\end{aligned}
$$

as well as the previously conjectured algebra [17] of the BRST charges themselves:

$$
\begin{aligned}
{\left[\hat{Q}_{j}(\sigma), \hat{Q}_{l}(\sigma)\right]_{+} } & =0, \forall j, l, \sigma \\
\hat{Q}_{j}^{2}(\sigma) & =0 .
\end{aligned}
$$

The extended BRST algebra in eq. (5.3a) follows directly from eq. (5.2c), and comprises one of the central results of this paper. 
In addition to the implied nilpotency (5.3b) of each BRST charge, I note the following two consistency checks among the algebras (5.2), (5.3), (4.3c), (4.6) and (4.7b). Both checks involve using eq. (5.2b) at $j=l$ as a definition of the total orbifold Virasoro generators $\left\{\hat{L}_{\hat{l} j}^{t}\right\}$. First, adding to this the BRST algebra (5.3a), we find that the algebra (5.2a) is implied. Second, an independent derivation of the total orbifold Virasoro algebra (4.3c) is obtained as follows: Start on the left side of eq. (4.3c) and use (5.2b) for one of the total Virasoro generators in the commutator. Then sequential application of the algebras (5.2b), (4.6), (4.7b) and a final application of eq. (4.2b) gives the right side of eq. (4.3c). These are of course generalizations of the consistency checks of the ordinary, untwisted BRST system (see e.g. ref. [23]) and the twisted BRST system of $\hat{c}=52$ matter [17].

\section{The physical states of cycle $j$ in sector $\sigma$}

For each sector $\sigma$ of each orbifold of permutation-type, we use the BRST charges (5.1) to define the physical states $\left\{|\chi(\sigma)\rangle_{j}\right\}$ of cycle $j$ as follows:

$$
\begin{aligned}
\hat{Q}_{j}(\sigma)|\chi(\sigma)\rangle_{j} & =0 \\
\hat{b}_{\hat{j} j}\left(\left(m+\frac{\hat{j}}{f_{j}(\sigma)}\right) \geq 0\right)|\chi(\sigma)\rangle_{j} & =0 \\
\hat{c}_{\hat{j} j}\left(\left(m+\frac{\hat{j}}{f_{j}(\sigma)}\right)>0\right)|\chi(\sigma)\rangle_{j} & =0 \\
\overline{\hat{j}}^{\prime}=0, \ldots, f_{j}(\sigma)-1, \quad j & =0, \ldots, N(\sigma)-1, \quad \sum_{j} f_{j}(\sigma)=K .
\end{aligned}
$$

Using then eqs. (5.2b) and (6.1a), (6.1b), we find also that the physical states are also annihilated by the non-negative modes of the total orbifold Virasoro generators

$$
\hat{L}_{\hat{j} j}^{t}\left(\left(m+\frac{\hat{j}}{f_{j}(\sigma)}\right) \geq 0\right)|\chi(\sigma)\rangle_{j}=0
$$

which is consistent because these generators have zero central extension. The role of the $\hat{c}$-condition in eq. (6.1c) will be noted in the following section.

Summing on the cycles, we find that the physical states are also annihilated by the total BRST charge of sector $\sigma$ :

$$
\begin{array}{rlrl}
\hat{Q}(\sigma) & \equiv \sum_{j} \hat{Q}_{j}(\sigma), & \hat{Q}^{2}(\sigma) & =0 \\
\hat{Q}(\sigma)|\chi(\sigma)\rangle & =0, & |\chi(\sigma)\rangle \equiv \otimes_{j}|\chi(\sigma)\rangle_{j} .
\end{array}
$$

Owing to the relations (5.3a), this condition for the full sector is equivalent to the BRST conditions for each of its cycles in eq. (6.1a). 


\section{$7 \quad$ Mode-ordered form of the twisted ghost systems}

To further analyze the physical states, we need more explicit forms of the operators in the twisted ghost systems. For this purpose, I define the following mode normal-ordered product

$$
\begin{aligned}
: \hat{A}_{\hat{j} j}\left(m+\frac{\hat{j}}{f_{j}(\sigma)}\right) \hat{B}_{\hat{l} l}\left(n+\frac{\hat{l}}{f_{l}(\sigma)}\right):_{M} \\
\equiv-\theta\left(\left(m+\frac{\hat{j}}{f_{j}(\sigma)}\right)>0\right) \hat{B}_{\hat{l} l}\left(n+\frac{\hat{l}}{f_{l}(\sigma)}\right) \hat{A}_{\hat{j} j}\left(m+\frac{\hat{j}}{f_{j}(\sigma)}\right) \\
\quad+\frac{1}{2} \delta_{m+\frac{\hat{j}}{f_{j}(\sigma)}, 0}\left[\hat{A}_{0 j}(0), \hat{B}_{\hat{l} l}\left(m+\frac{\hat{l}}{f_{l}(\sigma)}\right)\right] \\
\quad+\theta\left(\left(m+\frac{\hat{j}}{f_{j}(\sigma)}\right)<0\right) \hat{A}_{\hat{j} j}\left(m+\frac{\hat{j}}{f_{j}(\sigma)}\right) \hat{B}_{\hat{l} l}\left(n+\frac{\hat{l}}{f_{l}(\sigma)}\right)
\end{aligned}
$$

where $\hat{A}$ and $\hat{B}$ can be either $\hat{c}$ or $\hat{b}$.

Then the mode expansions in eq. (4.1) straightforwardly give the following relation between the operator-product normal-ordered quadratics and the mode normal-ordered quadratics

$$
\begin{aligned}
& : \hat{\bar{\psi}}_{\hat{j} j}(z, \sigma) \hat{\psi}_{\hat{l l}}(w, \sigma):=: \hat{\bar{\psi}}_{\hat{j} j}(z, \sigma) \hat{\psi}_{\hat{l} l}(w, \sigma):_{M}+\delta_{j l} \delta_{\hat{j}+\hat{l}, 0 \bmod f_{j}(\sigma)}\left(\hat{\Delta}_{\bar{j} j}(z, w)-\frac{1}{z-w}\right) \\
& \hat{\Delta}_{\overline{\hat{j} j}}(z, w) \equiv\left(\frac{z}{w}\right)^{2-\frac{\overline{\hat{j}}}{f_{j}(\sigma)}} \frac{1}{z-w}-\frac{z}{2 w^{2}} \delta_{\overline{\hat{j}}, 0} \\
& =\frac{1}{z-w}+\hat{a}_{\overline{\hat{j} j}}^{(0)}(w)+(z-w) \hat{a}_{\overline{\hat{j} j}}^{(1)}(w)+0(z-w)^{2} \\
& \hat{a}_{\overline{\hat{j} j}}^{(0)}(w) \equiv \frac{1}{w}\left(2-\frac{\overline{\hat{j}}}{f_{j}(\sigma)}-\frac{1}{2} \delta_{\overline{\hat{j}}, 0}\right) \\
& \hat{a}_{\overline{\hat{j} j}}^{(1)}(w) \equiv \frac{1}{2 w^{2}}\left\{\left(2-\frac{\overline{\hat{j}}}{f_{j}(\sigma)}\right)\left(1-\frac{\overline{\hat{j}}}{f_{j}(\sigma)}\right)-\delta_{\overline{\hat{j}}, 0}\right\}
\end{aligned}
$$

where $\overline{\hat{j}}=0,1, \ldots, f_{j}(\sigma)-1$ is again the pullback of $\hat{j}$ to the fundamental region. Comparing to the exact expression

$$
\hat{\Delta}_{0 j}(z, w)=\frac{z(z+w)}{2 w^{2}(z-w)}
$$

we see that the terms given explicitly in the Laurent expansion above are exact for $\overline{\hat{j}}=0$.

Eq. (7.2) then implies the local relations

$$
\begin{aligned}
& : \hat{\bar{\psi}}_{\hat{j} j}(z, \sigma) \hat{\psi}_{\hat{l l}}(z, \sigma):=: \hat{\bar{\psi}}_{\hat{j} j}(z, \sigma) \hat{\psi}_{\hat{l} l}(z, \sigma):_{M}+\delta_{j l} \delta_{\hat{j}+\hat{l}, 0 \bmod f_{j}(\sigma)} \hat{a}_{\hat{j} j}^{(0)}(z) \\
& : \hat{\bar{\psi}}_{\hat{j} j}(z, \sigma) \partial_{z} \hat{\psi}_{\hat{l l}}(z, \sigma):=: \hat{\bar{\psi}}_{\hat{j} j}(z, \sigma) \partial_{z} \hat{\psi}_{\hat{l} l}(z, \sigma): M+\delta_{j l} \delta_{\hat{j}+\hat{l}, 0 \bmod f_{j}(\sigma)}\left(\partial_{z} \hat{a}_{\hat{\bar{j} j}}^{(0)}(z)-\hat{a}_{\hat{j} j}^{(1)}(z)\right) \\
& : \partial_{z} \hat{\bar{\psi}}_{\hat{j} j}(z, \sigma) \hat{\psi}_{\hat{l} l}(z, \sigma):=: \partial_{z} \hat{\bar{\psi}}_{\hat{j} j}(z, \sigma) \hat{\psi}_{\hat{l} l}(z, \sigma):_{M}+\delta_{j l} \delta_{\hat{j}+\hat{l}, 0 \bmod f_{j}(\sigma)} \hat{a}_{\hat{j} j}^{(1)}(z)
\end{aligned}
$$


which are sufficient to obtain the mode-ordered forms of the relevant operators of the ghost system.

We begin with the mode-ordered form of the twisted ghost currents $\left\{\hat{J}^{G}\right\}$ :

$$
\begin{aligned}
\hat{J}_{\hat{j} j}^{G}(z, \sigma) & \equiv \hat{J}_{\hat{j} j}(z, \sigma)-\frac{3 f_{j}(\sigma)}{2 z} \delta_{\hat{j}, 0 \bmod f_{j}(\sigma)} \\
& =\sum_{\hat{l}=0}^{f_{j}(\sigma)-1}: \hat{\bar{\psi}}_{\hat{l} j}(z, \sigma) \hat{\psi}_{\hat{j}-\hat{l}, j}(z, \sigma): M \\
& =\sum_{m \in \mathbb{Z}} \hat{J}_{\hat{j} j}^{G}\left(m+\frac{\hat{j}}{f_{j}(\sigma)}\right) z^{-\left(m+\frac{\hat{j}}{f_{j}(\sigma)}\right)-1} \\
\hat{J}_{\hat{j} j}^{G}\left(m+\frac{\hat{j}}{f_{j}(\sigma)}\right) & =\hat{J}_{\hat{j} j}\left(m+\frac{\hat{j}}{f_{j}(\sigma)}\right)-\frac{3 f_{j}(\sigma)}{2} \delta_{m+\frac{\hat{j}}{f_{j}(\sigma)}, 0} \\
& =\sum_{\hat{l}=0}^{f_{j}(\sigma)-1} \sum_{p \in \mathbb{Z}}: \hat{c}_{\hat{l} j}\left(p+\frac{\hat{l}}{f_{j}(\sigma)}\right) \hat{b}_{\hat{j}-\hat{l}, j}\left(m-p+\frac{\hat{j}-\hat{l}}{f_{j}(\sigma)}\right):_{M} .
\end{aligned}
$$

In what follows we use only these mode-ordered forms $\left\{\hat{J}^{G}\right\}$ as the properly-ordered ghost currents. Since they differ from the original ghost currents $\{\hat{J}\}$ only by a $c$-number shift, we may in fact read $\hat{J} \rightarrow \hat{J}^{G}$ in all the mode algebras of section 4 . Using the definition (7.1) of the mode-ordering, we can for example write out the twisted ghost charge of cycle $j$ in full detail:

$$
\begin{aligned}
\hat{J}_{0 j}^{G}(m=0)= & \frac{1}{2}\left[\hat{c}_{0 j}(0), \hat{b}_{0 j}(0)\right]+\sum_{p=1}^{\infty}\left(\hat{c}_{0 j}(-p) \hat{b}_{0 j}(p)-\hat{b}_{0 j}(-p) \hat{c}_{0 j}(p)\right) \\
& -\sum_{\hat{l}=1}^{f_{j}(\sigma)-1} \hat{b}_{-\hat{l}, j}\left(-\frac{\hat{l}}{f_{j}(\sigma)}\right) \hat{c}_{\hat{l} j}\left(\frac{\hat{l}}{f_{j}(\sigma)}\right) \\
& +\sum_{\hat{l}=1}^{f_{j}(\sigma)-1} \sum_{p=1}^{\infty}\left\{\hat{c}_{\hat{l} j}\left(-p+\frac{\hat{l}}{f_{j}(\sigma)}\right) \hat{b}_{-\hat{l}, j}\left(p-\frac{\hat{l}}{f_{j}(\sigma)}\right)\right. \\
& \left.\quad-\hat{b}_{-\hat{l}_{, j}}\left(-p-\frac{\hat{l}}{f_{j}(\sigma)}\right) \hat{c}_{\hat{l} j}\left(p+\frac{\hat{l}}{f_{j}(\sigma)}\right)\right\} .
\end{aligned}
$$

Note that the terms involving the zero modes $\hat{c}_{0 j}(0)$ and $\hat{b}_{0 j}(0)$ of the twisted ghosts are isomorphic to the standard ghost-charge operator of ordinary (untwisted) BRST.

With eqs. (3.1b) and (7.2), we also obtain the mode-ordered form of the extended ghost stress-tensors and their orbifold Virasoro generators:

$$
\begin{aligned}
\hat{\theta}_{\hat{j} j}^{G}(z, \sigma)= & -\frac{\hat{a}_{f_{j}(\sigma)}}{z^{2}} \delta_{\hat{j}, 0 \bmod f_{j}(\sigma)} \\
& +\sum_{\hat{l}=0}^{f_{j}(\sigma)-1}:\left(\hat{\bar{\psi}}_{\hat{l} j}(z, \sigma) \partial_{z} \hat{\psi}_{\hat{j}-\hat{l}, j}(z, \sigma)+2 \partial_{z} \hat{\bar{\psi}}_{\hat{l} j}(z, \sigma) \hat{\psi}_{\hat{j}-\hat{l}, j}(z, \sigma)\right):_{M}
\end{aligned}
$$




$$
\begin{aligned}
& \hat{L}_{\hat{j} j}^{G}\left(m+\frac{\hat{j}}{f_{j}(\sigma)}\right)=-\hat{a}_{f_{j}(\sigma)} \delta_{m+\frac{\hat{j}}{f_{j}(\sigma)}, 0} \\
&-\sum_{\hat{l}=0}^{f_{j}(\sigma)-1} \sum_{p \in \mathbb{Z}}\left(m+p+\frac{\hat{j}+\hat{l}}{f_{j}(\sigma)}\right): \hat{c}_{\hat{l} j}\left(p+\frac{\hat{l}}{f_{j}(\sigma)}\right) \hat{b}_{\hat{j}-\hat{l}, j}\left(m-p+\frac{\hat{j}-\hat{l}}{f_{j}(\sigma)}\right): M \\
& \hat{a}_{f_{j}(\sigma)} \equiv \frac{13 f_{j}^{2}(\sigma)-1}{12 f_{j}(\sigma)} .
\end{aligned}
$$

In what follows, I shall refer to the quantity $\hat{a}_{f_{j}(\sigma)}$ in eq. $(7.7 \mathrm{c})$ as the intercept of cycle $j$ in each sector $\sigma$. Mode-ordered expressions can also be obtained for the twisted BRST currents $\left\{\hat{J}_{\hat{j} j}^{G}\right\}$ and the BRST charges $\left\{\hat{Q}_{j}\right\}$, but these will not be needed in the present development.

As an application of the mode-ordered forms (7.5b) and (7.7b), we may use both the $\hat{b}$ and the $\hat{c}$ conditions (6.1b), (6.1c) on the physical states of cycle $j$ to compute directly that

$$
\begin{array}{r}
\left(\hat{J}_{\hat{j} j}^{G}\left(\left(m+\frac{\hat{j}}{f_{j}(\sigma)}\right) \geq 0\right)+\frac{1}{2} \delta_{m+\frac{\hat{j}}{f_{j}(\sigma)}, 0}\right)|\chi(\sigma)\rangle_{j}=0 \\
\left(\hat{L}_{\hat{j} j}^{G}\left(\left(m+\frac{\hat{j}}{f_{j}(\sigma)}\right) \geq 0\right)+\hat{a}_{f_{j}(\sigma) \delta_{m+\frac{\hat{j}}{f_{j}(\sigma)}, 0}}\right)|\chi(\sigma)\rangle_{j}=0 .
\end{array}
$$

We emphasize that these results require attention to the definition (7.1) of mode-ordering. In particular, the explicit form (7.6) of the twisted ghost charge is needed to see that the physical states of cycle $j$ have ghost number $\left(-\frac{1}{2}\right)$, uniformly, as in ordinary untwisted BRST (see e.g. ref. [25]).

For completeness, we finally give the action on the physical states of the ghost current modes and ghost Virasoro generators of sector $\sigma$

$$
\begin{gathered}
\hat{J}_{\sigma}^{G}(m) \equiv \sum_{j} \hat{J}_{0 j}^{G}(m), \quad \hat{L}_{\sigma}^{G}(m) \equiv \sum_{j} \hat{L}_{0 j}^{G}(m) \\
\left(\hat{J}_{\sigma}^{G}(m \geq 0)+\frac{N(\sigma)}{2} \delta_{m, 0}\right)|\chi(\sigma)\rangle=0 \\
\left(\hat{L}_{\sigma}^{G}(m \geq 0)+\frac{1}{12}\left(13 K-\sum_{j} \frac{1}{f_{j}(\sigma)}\right) \delta_{m, 0}\right)|\chi(\sigma)\rangle=0
\end{gathered}
$$

where $\left\{L_{\sigma}^{G}(m)\right\}$ satisfies the Virasoro algebra (4.5c) at $\hat{c}^{G}(\sigma)=-26 K, N(\sigma)$ is the number of cycles in sector $\sigma$ and the product states $\{|\chi(\sigma)\rangle\}$ are defined in eq. (6.3b).

\section{The extended physical-state conditions}

The final step in this paper is the transition from the action of the ghost operators to that of the matter operators on the physical states.

This transition is now effected in a single step, using eq. (7.8b) and the fact (6.2) that the physical states are annihilated by the non-negative modes of the total orbifold 


\begin{tabular}{|c|c|c|}
\hline$f_{j}(\sigma)$ & $\hat{c}_{j}(\sigma)$ & $\hat{a}_{f_{j}(\sigma)}$ \\
\hline 1 & 26 & 1 \\
2 & 52 & $17 / 8$ \\
3 & 78 & $29 / 9$ \\
4 & 104 & $69 / 16$ \\
5 & 130 & $27 / 5$ \\
6 & 156 & $155 / 24$ \\
\hline
\end{tabular}

Table 1. Numerical values.

Virasoro generators $\left\{\hat{L}^{t}\right\}$ with $\hat{c}_{j}^{t}(\sigma)=0$. The result is the following extended physicalstate conditions of cycle $j$ in sector $\sigma$ :

$$
\begin{gathered}
\left(\hat{L}_{\hat{j} j}\left(\left(m+\frac{\hat{j}}{f_{j}(\sigma)}\right) \geq 0\right)-\hat{a}_{f_{j}(\sigma)} \delta_{m+\frac{\hat{j}}{f_{j}(\sigma)}, 0}\right)|\chi(\sigma)\rangle_{j}=0 \\
{\left[\hat{L}_{\hat{j} j}\left(m+\frac{\hat{j}}{f_{j}(\sigma)}\right), \hat{L}_{\hat{l} l}\left(l+\frac{\hat{l}}{f_{l}(\sigma)}\right)\right]} \\
=\delta_{j l}\left\{\left(m-n+\frac{\hat{j}-\hat{l}}{f_{j}(\sigma)}\right) \hat{L}_{\hat{j}+\hat{l}, j}\left(m+n+\frac{\hat{j}+\hat{l}}{f_{j}(\sigma)}\right)\right. \\
\left.\quad+\frac{\hat{c}_{j}(\sigma)}{12}\left(m+\frac{\hat{j}}{f_{j}(\sigma)}\right)\left(\left(m+\frac{\hat{j}}{f_{j}(\sigma)}\right)^{2}-1\right) \delta_{m+n+\frac{\hat{j}+\hat{l}}{f_{j}(\sigma)}, 0}\right\} \\
\hat{c}_{j}(\sigma)=26 f_{j}(\sigma), \quad \hat{a}_{f_{j}(\sigma)}=\frac{13 f_{j}^{2}(\sigma)-1}{12 f_{j}(\sigma)} \\
\overline{\hat{j}}=0,1, \ldots, f_{j}(\sigma)-1, \quad j=0,1, \ldots, N(\sigma)-1, \quad \sum_{j} f_{j}(\sigma)=K .
\end{gathered}
$$

These conditions on the twisted matter of the new string theories are a central result of the paper. We remind that the extended Virasoro algebra (8.1b) of each cycle $j$ is called an orbifold Virasoro algebra of order $f_{j}(\sigma)$ at cycle central charge $\hat{c}_{j}(\sigma)=26 f_{j}(\sigma)$, and the intercept $\hat{a}_{f_{j}(\sigma)}$ of cycle $j$ in sector $\sigma$ descends directly from eq. (7.8b) of the ghost system.

The extended physical state conditions (8.1a) are the operator analogues of the classical extended Virasoro constraints

$$
\hat{\theta}_{\hat{j} j}(z, \sigma)=\hat{L}_{\hat{j} j}\left(m+\frac{\hat{j}}{f_{j}(\sigma)}\right)=0, \forall \hat{j} j \text { in sector } \sigma
$$

associated to the general permutation gravities of the extended actions given for these theories in ref. [16]. Further remarks on the action formulations are included in appendix B.

In this sense the cycle central charges $\left\{\hat{c}_{j}(\sigma)\right\}$ and the cycle-intercepts $\left\{\hat{a}_{f_{j}(\sigma)}\right\}$ are the fundamental numbers obtained in our BRST quantization, and both quantities are universal in that they depend only on the length $f_{j}(\sigma)$ of cycle $j$ in sector $\sigma$. The preceding table of numerical values underscores that both quantities increase monotonically with cycle-length. 
Using the cycle basis of each element $\omega\left(h_{\sigma}\right) \in H(\text { perm })_{K}$, the twisted matter of each sector $\sigma$ of each orbifold-string of permutation-type is described by cycle-collections of our result in eq. (8.1). I illustrate this here with some simple examples.

The first case in the table $f_{j}(\sigma)=1$ includes ordinary untwisted critical string theory. Copies of ordinary string theory are encountered here for example in the untwisted sectors $\sigma=0$ (the trivial element of $H(\text { perm })_{K}$ and $H_{26}^{\prime}$ ) of all the orbifold-string theories of permutation-type:

$$
\begin{aligned}
\left(\hat{L}_{0 j}(m \geq 0)-\delta_{m, 0}\right)|\chi(0)\rangle_{j} & =0 \\
{\left[\hat{L}_{0 j}(m), \hat{L}_{0 l}(n)\right] } & =\delta_{j l}\left((m-n) L_{0 j}(m+n)+\frac{\hat{c}_{j}(0)}{12} m\left(m^{2}-1\right) \delta_{m+n, 0}\right) \\
\hat{c}_{j}(0)=26, \quad N(0) & =K, \quad \overline{\hat{j}}=0, \quad j=0,1, \ldots, K-1 .
\end{aligned}
$$

With a set of right-mover copies and the relabeling $j \rightarrow I$, this is recognized as the physicalstate conditions of the original $K$ copies $\mathrm{U}(1)^{26 K}$ of the ordinary untwisted closed string $\mathrm{U}(1)^{26}$, i.e., the starting point of the orbifold program in this case. There are no cycles of unit length in the non-trivial twisted sectors $\sigma=1, \ldots, K-1$ of $H(\text { perm })_{K}=\mathbb{Z}_{K}$, but cycles of unit length occur frequently in the elements of $H(\text { perm })_{K}=S_{K}$ - where they also describe unit-cycle strings with $\hat{c}_{j}(\sigma)=26$ and unit intercept. We emphasize however that these unit-cycle strings are not ordinary strings for non-trivial elements of the space-time automorphism group $H_{26}^{\prime}$. Indeed, for example, each sector $\sigma$ of the ordinary space-time orbifolds $\mathrm{U}(1)^{26} / H_{26}^{\prime}$ (the closed-string sectors of each orientation-orbifold string system) is also described by the ordinary physical-state condition (8.3) at $K=1$.

The simplest example of the second case $f_{j}(\sigma)=2$ is the single twisted sector $\sigma=1$ of $H(\text { perm })_{2}=\mathbb{Z}_{2}$ or $\mathbb{Z}_{2}$ (w.s. $)$, where we find

$$
\begin{aligned}
& \quad\left(\hat{L}_{\hat{j} 0}\left(m+\frac{\hat{j}}{2}\right)-\frac{17}{8} \delta_{m+\frac{\hat{j}}{2}, 0}\right)|\chi(1)\rangle_{0}=0 \\
& {\left[\hat{L}_{\hat{j} 0}\left(m+\frac{\hat{j}}{2}\right), \hat{L}_{\hat{l} 0}\left(n+\frac{\hat{l}}{2}\right)\right]} \\
& =\left(m-n+\frac{\hat{j}-\hat{l}}{2}\right) \hat{L}_{\hat{j}+\hat{l}, 0}\left(m+n+\frac{\hat{j}+\hat{l}}{2}\right) \\
& \quad+\frac{52}{12}\left(m+\frac{\hat{j}}{2}\right)\left(\left(m+\frac{\hat{j}}{2}\right)^{2}-1\right) \delta_{m+n+\frac{\hat{j}+\hat{l}}{2}, 0} \\
& \hat{c}(1)=\hat{c}_{0}(1)=52, \quad N(1)=1, \quad j=0, \quad \overline{\hat{j}}=0,1 .
\end{aligned}
$$

With the relabeling $\overline{\hat{j}} \rightarrow \bar{u}=0,1$, this collection is recognized as the two extended physicalstate conditions of twisted $\hat{c}=52$ matter in refs. [16-20]. These systems appear for example in the twisted open-string sectors of the orientation-orbifold string theories [15-19] or, adding right-mover copies, in the generalized $\mathbb{Z}_{2}$-permutation orbifolds $[15-18,20]$. 
The result (8.4) is included in the extended physical-state conditions for the twisted sectors $\sigma=1, \ldots, K-1$ of $\mathbb{Z}_{K}, K=$ prime, where $f_{j}(\sigma)=K$ for each sector:

$$
\begin{aligned}
& \quad\left(\hat{L}_{\hat{j} 0}\left(\left(m+\frac{\hat{j}}{K}\right) \geq 0\right)-\hat{a}_{K} \delta_{m+\frac{\hat{j}}{K}, 0}\right)|\chi(\sigma)\rangle_{0}=0 \\
& {\left[\hat{L}_{\hat{j} 0}\left(m+\frac{\hat{j}}{K}\right), \hat{L}_{\hat{l} 0}\left(n+\frac{\hat{l}}{K}\right)\right]} \\
& =\left(m-n+\frac{\hat{j}-\hat{l}}{K}\right) \hat{L}_{\hat{j}+\hat{l}, 0}\left(m+n+\frac{\hat{j}+\hat{l}}{K}\right) \\
& \quad+\frac{\hat{c}_{0}(\sigma)}{12}\left(m+\frac{\hat{j}}{K}\right)\left(\left(m+\frac{\hat{j}}{K}\right)^{2}-1\right) \delta_{m+n+\frac{\hat{j}+\hat{l}}{K}, 0} \\
& \hat{c}_{0}(\sigma)=26 K, \quad \hat{a}_{K}=\frac{13 K^{2}-1}{12 K}, \quad K=\text { prime } \\
& N(\sigma)=1, \quad j=0, \quad \overline{\hat{j}}^{2}=0,1, \ldots, K-1, \quad \sigma=1, \ldots, K-1 .
\end{aligned}
$$

The extended physical-state conditions for prime cyclic groups were conjectured in ref. [18] and verified at the interacting level in ref. [20].

The cycle-bases for the $K-1$ twisted sectors of the general cyclic group $H(\text { perm })_{K}=\mathbb{Z}_{K}$

$$
\begin{aligned}
& \overline{\hat{j}}=0,1, \ldots, f_{j}(\sigma)-1, \quad j=0,1, \ldots, \frac{K}{f_{j}(\sigma)}-1, \quad \sum_{j} f_{j}(\sigma)=K \\
& \sigma=1, \ldots, K-1
\end{aligned}
$$

were described in eq. (2.7). Then for example we find the following extended physicalstate conditions for the three twisted sectors $\sigma=1,2,3$ of $H(\text { perm })_{4}=\mathbb{Z}_{4}$ : There are two single-cycle sectors with length 4

$$
\begin{gathered}
\left(\hat{L}_{\hat{j} 0}\left(\left(m+\frac{\hat{j}}{4}\right) \geq 0\right)-\frac{69}{16} \delta_{m+\frac{\hat{j}}{4}, 0}\right)|\chi(\sigma)\rangle_{0}=0 \\
\quad\left[\hat{L}_{\hat{j} 0}\left(m+\frac{\hat{j}}{4}\right), \hat{L}_{\hat{l} 0}\left(n+\frac{\hat{l}}{4}\right)\right] \\
=\left(m-n+\frac{\hat{j}-\hat{l}}{4}\right) \hat{L}_{\hat{j}+\hat{l}, 0}\left(m+n+\frac{\hat{j}+\hat{l}}{4}\right) \\
\quad+\frac{104}{12}\left(m+\frac{\hat{j}}{4}\right)\left(\left(m+\frac{\hat{j}}{4}\right)^{2}-1\right) \delta_{m+n+\frac{\hat{j}+\hat{l}}{4}, 0} \\
j=0, \quad \overline{\hat{j}}=0,1,2,3, \quad \sigma=1,3
\end{gathered}
$$

and another sector with two cycles of length 2

$$
\left(\hat{L}_{\hat{j} j}\left(\left(m+\frac{\hat{j}}{2}\right) \geq 0\right)-\frac{17}{8} \delta_{m+\frac{\hat{j}}{2}, 0}\right)|\chi(2)\rangle_{j}=0
$$




$$
\begin{aligned}
& {\left[\hat{L}_{\hat{j} j}\left(m+\frac{\hat{j}}{2}\right), \hat{L}_{\hat{l} l}\left(n+\frac{\hat{l}}{2}\right)\right]} \\
& =\delta_{j l}\left\{\left(m-n+\frac{\hat{j}-\hat{l}}{2}\right) \hat{L}_{\hat{j}-\hat{l}, j}\left(m+n+\frac{\hat{j}+\hat{l}}{2}\right)\right. \\
& \left.\quad+\frac{52}{12}\left(m+\frac{\hat{j}}{2}\right)\left(\left(m+\frac{\hat{j}}{2}\right)^{2}-1\right) \delta_{m+n+\frac{\hat{j}+\hat{l}}{2}, 0}\right\} \\
& j=0,1, \quad \overline{\hat{j}}=0,1, \quad \sigma=2 .
\end{aligned}
$$

For $H$ (perm $)_{8}=\mathbb{Z}_{8}$, one has a single-cycle sector with length 8 , five sectors with two cycles of length 4 each, and one sector with four cycles of length 2. Further cyclic examples are left to the reader, and we remind that the cycle-bases for the permutation groups $H(\text { perm })_{K}=S_{K}$ are given in eq. (2.6).

Finally, we mention the physical-state condition of any sector $\sigma$ (see eq. (4.5))

$$
\begin{array}{r}
\hat{L}_{\sigma}(m)=\sum_{j} \hat{L}_{0 j}(m) \\
\left(\hat{L}_{\sigma}(m \geq 0)-\frac{\delta_{m, 0}}{12}\left(13 K-\sum_{j} \frac{1}{f_{j}(\sigma)}\right)\right)|\chi(\sigma)\rangle=0 \\
{\left[\hat{L}_{\sigma}(m), \hat{L}_{\sigma}(n)\right]=(m-n) \hat{L}_{\sigma}(m+n)+\frac{26 K}{12} m\left(m^{2}-1\right) \delta_{m+n, 0}}
\end{array}
$$

where $\left\{\hat{L}_{\sigma}\right\}$ are the physical Virasoro generators of sector $\sigma$ for any $H(\text { perm })_{K}$ and the product-states $\{|\chi(\sigma)\rangle\}$ are defined in eq. (6.3b). With the sum rule in eq. (8.1d), this condition follows simply from our central result (8.1) by summing over the cycles of each sector.

\section{Conclusions}

We have completed a BRST quantization of the bosonic prototypes of the generalized permutation-orbifold string theories and their open-string analogues

$$
\frac{\mathrm{U}(1)^{26 K}}{H_{+}}, \quad\left[\frac{\mathrm{U}(1)^{26 K}}{H_{+}}\right]_{\text {open }}, \quad H_{+} \subset H(\text { perm })_{K} \times H_{26}^{\prime}
$$

where $H_{26}^{\prime}$ is any automorphism group of the critical closed string $\mathrm{U}(1)^{26}$ and $H(\text { perm })_{K}$ is any permutation group on the $K$ copies. The matter of each sector $\sigma$ of these orbifolds lives at sector central charge $\hat{c}(\sigma)=26 K$. We remind that the orientation-orbifold string systems

$$
\frac{\mathrm{U}(1)^{26}}{H_{-}}=\frac{\mathrm{U}(1)_{L}^{26} \times \mathrm{U}(1)_{R}^{26}}{H_{-}}, \quad H_{-} \subset \mathbb{Z}_{2}(w . s .) \times H_{26}^{\prime}
$$

are also described in our quantization: The twisted open-string sectors of these theories are included in the open-string analogues at $K=2$ and $\hat{c}(\sigma)=52$, while the twisted closedstring sectors are the ordinary space-time orbifolds $\mathrm{U}(1)^{26} / H_{26}^{\prime}$ at $\hat{c}(\sigma)=26$. These last 
cases correspond to the trivial element of $\mathbb{Z}_{2}(w . s$.$) - and hence ordinary physical-state$ conditions for each sector. The extended action formulations [16] of the bosonic prototypes are reviewed in appendix B, which also points out a generalization of the orientationorbifold string systems.

Using the cycle-bases of general permutation groups, our central result in this paper is the construction of one twisted BRST system per cycle per sector in each of the orbifoldstring systems (9.1) and (9.2). In particular, we have found the extended BRST algebra (5.3a), conjectured in ref. [17], which gives one nilpotent BRST charge $\hat{Q}_{j}(\sigma)$ for each cycle $j$ in each sector $\sigma$ of these orbifolds. The twisted BRST systems then imply the extended physical state conditions (8.1) for the matter at cycle central charge $\hat{c}_{j}(\sigma)=26 f_{j}(\sigma)$, where $f_{j}(\sigma)$ is the length of cycle $j$. The sector central charges of the matter are recovered as the cycle sums $\hat{c}(\sigma)=\sum_{j} \hat{c}_{j}(\sigma)=26 K$. The extended physical-state conditions also exhibit another set of fundamental numbers, the so-called cycle-intercepts $\hat{a}_{f_{j}(\sigma)}$ in eq. (8.1c). We remind that a right-mover copy of the results given here are necessary to describe the twisted closed-string sectors of these orbifolds.

Our results here therefore generalize the construction in ref. [17] of one twisted BRST system for the non-trivial element of $H(\text { perm })_{2}=\mathbb{Z}_{2}$ or $\mathbb{Z}_{2}$ (w.s.), with single-cycle length $f_{0}(1)=2$ and matter central charge $\hat{c}_{0}(1)=52$. We emphasize that all aspects of our new twisted BRST systems, including the extended physical-state conditions, are separable in the cycles $j$ of each sector. This is in accord with the extended actions [16] of these theories (see appendix B), and with earlier work on the WZW permutation orbifolds [9] with trivial $H_{26}^{\prime}$.

In this paper, the generators $\left\{\hat{L}_{\hat{j} j}\left(m+\frac{\hat{j}}{f_{j}(\sigma)}\right)\right\}$ of the orbifold Virasoro algebras of the matter have been treated abstractly, so our first task in the next paper of this series will be the explicit construction of these generators in terms of the twisted matter fields. It is in this step that each element of the automorphism group $H_{26}^{\prime}$ is encoded. With this information, we will also find the equivalent, reduced formulation of the physical-state problem of each cycle $j$ at reduced cycle-central charge $c_{j}(\sigma)=26$, and begin our study of the target space-times of the orbifold-string theories of permutation-type.

\section{A Fermi statistics for $\hat{c}$ 's}

For any numerical function $\mathcal{O}$, define the quadratic inner product

$$
(\hat{c}, \mathcal{O} \hat{c})_{\hat{j} j}(m) \equiv \sum_{\hat{l}=0}^{f_{j}(\sigma)-1} \sum_{p \in \mathbb{Z}} \hat{c}_{\hat{l} j}\left(p+\frac{\hat{l}}{f_{j}(\sigma)}\right) \hat{c}_{\hat{j}-\hat{l}, j}\left(m-p+\frac{\hat{j}-\hat{l}}{f_{j}(\sigma)}\right) \mathcal{O}
$$

on the twisted ghost modes $\hat{c}$. Then the vanishing anticommutator (4.2b) of any two $\hat{c}$ 's gives the following list of equivalences among various pairs of $\mathcal{O}$ 's

$$
\begin{aligned}
1 & \approx 0 \\
p^{2} & \approx m p \\
\left(p+\frac{\hat{l}}{f_{j}(\sigma)}\right)^{2} & \approx\left(m+\frac{\hat{j}}{f_{j}(\sigma)}\right)\left(p+\frac{\hat{l}}{f_{j}(\sigma)}\right)
\end{aligned}
$$

in this inner product, and these equivalences were used to simplify the right-side of eq. (4.8c). 


\section{B Extended actions and generalized orientation orbifolds}

Ref. [16] gave the extended actions for the $\hat{c}=52$ twisted open-string sectors of the orientation-orbifold string systems

$$
\begin{aligned}
& \frac{\mathrm{U}(1)_{L}^{26} \times \mathrm{U}(1)_{R}^{26}}{H_{-}}, \quad H_{-} \subset \mathbb{Z}_{2}(w . s .) \times H_{26}^{\prime} \\
& \hat{S}_{\sigma}=\frac{1}{4 \pi} \int d t \int_{0}^{\pi} d \xi \sum_{u, v, w=0}^{1} \hat{h}_{(u+v+w)}^{m n} \hat{H}^{(u)} \partial_{m} \hat{X}^{n(r) \mu v} \mathcal{G}_{n(r) \mu ; n(s) \nu}(\sigma) \partial_{m} \hat{X}^{n(s) \nu w} .
\end{aligned}
$$

Here $\hat{h}_{(u)}^{m n}$ is the inverse of $\hat{h}_{m n}^{(u)}$, which is the twisted metric of $\mathbb{Z}_{2}$-permutation gravity associated to the world-sheet orientation-reversing element of $\mathbb{Z}_{2}$ (w.s.). The explicit forms of the extended diffeomorphisms, the densities $\hat{H}^{(u)}$ and the twisted metric $\mathcal{G}(\sigma)$ of $H_{26}^{\prime}$ are also given there, as well as the branes of these strings. The generators $\left\{\hat{L}_{u}\left(m+\frac{u}{2}\right)\right\}$ of the orbifold Virasoro algebras are singly-twisted and the matter currents $\left\{\hat{J}\left(m+\frac{n(r)}{\rho(\sigma)}+\frac{u}{2}\right)\right\}$ are doubly-twisted, where $\{n(r) / \rho(\sigma)\}$ are the spectral fractions of the elements of $H_{26}^{\prime}$. The twisted $\hat{c}=26$ closed-string sectors of the orientation orbifold form the ordinary spacetime orbifold $\mathrm{U}(1)^{26} / H_{26}^{\prime}$, with ordinary Polyakov gravity, ordinary Virasoro algebras and singly-twisted currents $\left\{\hat{J}\left(m+\frac{n(r)}{\rho(\sigma)}\right)\right\}$.

Ref. [16] also gave the extended actions of each twisted $\hat{c}=26 K$ closed-string sector of the generalized permutation orbifolds

$$
\begin{aligned}
\frac{\mathrm{U}(1)^{26 K}}{H_{+}}, & H_{+} \subset H(\text { perm })_{K} \times H_{26}^{\prime} \\
\hat{S}_{\sigma}= & \frac{1}{8 \pi} \int d t \int_{0}^{2 \pi} d \xi \sum_{j} f_{j}(\sigma) \sum_{\hat{j}, \hat{h}, \hat{l}=0}^{f_{j}(\sigma)-1} \times \\
& \times \hat{h}_{(\hat{j}+\hat{k}+\hat{l}) j}^{m n} \hat{H}^{(\hat{j}) j} \partial_{m} \hat{X}^{n(r) \mu \hat{k} j} \mathcal{G}_{n(r) \mu ; n(s) \nu}(\sigma) \partial_{n} \hat{X}^{n(s) \nu \hat{l} j} \\
\sum_{j} f_{j}(\sigma)= & K
\end{aligned}
$$

where $h_{m n}^{(\hat{j}) j}$ is the extended metric of the permutation gravity associated to each equivalence class of $H(\text { perm })_{K}$. The monodromies, extended diffeomorphisms and the explicit form of the densities $\hat{H}^{(\hat{j}) j}$ in these sectors are also given in ref. [16]. Again, the generators $\left\{\hat{L}_{\hat{j} j}\left(m+\frac{\hat{j}}{f_{j}(\sigma)}\right)\right\}$ of the orbifold Virasoro algebra are singly-twisted and the matter currents $\left\{\hat{J}\left(m+\frac{n(r)}{\rho(\sigma)}+\frac{\hat{j}}{f_{j}(\sigma)}\right)\right\}$ are doubly-twisted. In these cases of course one obtains a left- and right-mover copy of each algebra.

On the basis of these results, one expects that the extended actions of the $\hat{c}=26 K$ open-string analogues of the generalized permutation orbifolds will have the same form as that shown in eq. (B.2), but with the substitution

$$
\left[\frac{\mathrm{U}(1)^{26 K}}{H_{+}}\right]_{\mathrm{open}}: \int_{0}^{2 \pi} d \xi \rightarrow \int_{0}^{\pi} d \xi .
$$


By construction these extended actions have the same bulk-invariances and modeing as those of the generalized permutation orbifolds, and these actions reduce for $K=f_{j}(\sigma)=2$ to the actions (B.1) of the open-string sectors of the orientation orbifolds. See also the construction of general twisted open strings in ref. [15].

Note that the extended actions (B.1)-(B.3) are separable in the cycle-label $j$, consistent with the results of the text and earlier work in refs. [9, 16-18]. In principle, the twisted BRST systems of this paper can also be derived from these actions by the Faddeev-Popov method.

In fact, there exist other sets of bosonic prototypes of the new string theories, for example the generalized orientation-orbifold string theories

$$
\frac{\left(\mathrm{U}(1)_{L}^{26} \times \mathrm{U}(1)_{R}^{26}\right)^{K}}{H_{-+}}, \quad H_{-+} \subset \mathbb{Z}_{2}(\text { w.s. }) \times H(\text { perm })_{K} \times H_{26}^{\prime}
$$

which reduce to the orientation orbifolds (B.1a) when $K=1$. Detailed construction of these theories for higher $K$ is beyond the scope of this paper, so I confine the discussion here to some preliminary remarks on their structure.

I begin with some simple properties of these theories as CFT's. Like orientation orbifolds, the presence of the world-sheet orientation group $\mathbb{Z}_{2}$ (w.s.) in the divisor tells us that the generalized orientation orbifolds have an equal number of twisted open- and closedstring sectors, now at sector central charges $\hat{c}=52 K$ and $26 K$ respectively. In fact, the closed-string sectors (corresponding to the trivial element of $\mathbb{Z}_{2}(w . s$.$) ) of these theories$ clearly form (two copies of) the generalized permutation orbifold (B.2a), while the sectors corresponding to the trivial element of $H$ (perm) $)_{K}$ comprise (K copies of) the ordinary orientation orbifold (B.1a).

It is also clear that the matter currents of the open-string sectors of these theories are triply-twisted, with three spectral fractions corresponding to the elements of $H_{26}^{\prime}, H(\text { perm })_{K}$ and $\mathbb{Z}_{2}$ (w.s.):

$$
\begin{aligned}
\hat{J}\left(m+\frac{n(r)}{\rho(\sigma)}+\frac{\hat{j}}{f_{j}(\sigma)}+\frac{u}{2}\right) & & \\
\bar{n}(r) \in[0,1, \ldots, \rho(\sigma)-1], & \bar{u} & =0,1 \\
\overline{\hat{j}}=0,1, \ldots, f_{j}(\sigma)-1, & j & =0,1, \ldots, N(\sigma)-1, \quad \sum_{j} f_{j}(\sigma)=K .
\end{aligned}
$$

Triply-twisted matter is a degree of complexity not yet studied in the orbifold program.

By the same token, the open-string sectors should exhibit new doubly-twisted orbifoldVirasoro generators of the form $\left\{\hat{L}_{\hat{j} j u}\left(m+\frac{\hat{j}}{f_{j}(\sigma)}+\frac{u}{2}\right)\right\}$, as well as doubly-twisted BRST systems $\left(\hat{c}_{\hat{j} j u}, \hat{b}_{\hat{j} j u}, \hat{J}_{\hat{j} j u}^{B}, \ldots\right)$ and open-string extended actions of the schematic form:

$$
\begin{aligned}
\hat{S}_{\sigma}=\frac{1}{8 \pi} \int d t \int_{0}^{\pi} & d \xi \sum_{j} f_{j}(\sigma) \sum_{\hat{j}, \hat{k}, \hat{l}=0}^{f_{j}(\sigma)-1} \sum_{u, v, w=0}^{1} \times \\
& \times \hat{h}_{(\hat{j}+\hat{k}+\hat{l}) j(u+v+w)}^{m n} \hat{H}^{(\hat{j}) j(u)} \partial_{m} \hat{X}^{n(r) \mu \hat{k} j v} \mathcal{G}_{n(r) \mu ; n(s) \nu}(\sigma) \partial_{n} \hat{X}^{n(s) \nu \hat{l} j w} .
\end{aligned}
$$


Here the quantities $\hat{h}_{m n}^{(\hat{j}) j(u)}$ and $\hat{H}^{(\hat{j}) j(u)}$ are the extended metric and density of the worldsheet permutation gravity associated with each equivalence class of the product permutation group $\mathbb{Z}_{2}($ w.s. $) \times H(\text { perm })_{K}$. I have not worked out these last structures in detail, and beyond this I will not speculate in this paper.

\section{Acknowledgments}

For helpful discussion and encouragement, I thank L. Alvarez-Gaumé, C. Bachas, J. de Boer, S. Frolov, O. Ganor, E. Kiritsis, A. Neveu, H. Nicolai, N. Obers, B. Pioline, M. Porrati, E. Rabinovici, V. Schomerus, C. Schweigert, M. Staudacher, R. Stora, C. Thorn, E. Verlinde and J.-B. Zuber.

Open Access. This article is distributed under the terms of the Creative Commons Attribution Noncommercial License which permits any noncommercial use, distribution, and reproduction in any medium, provided the original author(s) and source are credited.

\section{References}

[1] L. Borisov, M.B. Halpern and C. Schweigert, Systematic approach to cyclic orbifolds, Int. J. Mod. Phys. A 13 (1998) 125 [hep-th/9701061] [SPIRES].

[2] J. Evslin, M.B. Halpern and J.E. Wang, General Virasoro construction on orbifold affine algebra, Int. J. Mod. Phys. A 14 (1999) 4985 [hep-th/9904105] [SPIRES].

[3] J. de Boer, J. Evslin, M.B. Halpern and J.E. Wang, New duality transformations in orbifold theory, Int. J. Mod. Phys. A 15 (2000) 1297 [hep-th/9908187] [SPIRES].

[4] J. Evslin, M.B. Halpern and J.E. Wang, Cyclic coset orbifolds, Int. J. Mod. Phys. A 15 (2000) 3829 [hep-th/9912084] [SPIRES].

[5] M.B. Halpern and J.E. Wang, More about all current-algebraic orbifolds, Int. J. Mod. Phys. A 16 (2001) 97 [hep-th/0005187] [SPIRES].

[6] J. de Boer, M.B. Halpern and N.A. Obers, The operator algebra and twisted KZ equations of WZW orbifolds, JHEP 10 (2001) 011 [hep-th/0105305] [SPIRES].

[7] M.B. Halpern and N.A. Obers, Two large examples in orbifold theory: Abelian orbifolds and the charge conjugation orbifold on $\mathrm{SU}(N)$, Int. J. Mod. Phys. A 17 (2002) 3897 [hep-th/0203056] [SPIRES].

[8] M.B. Halpern and F. Wagner, The general coset orbifold action, Int. J. Mod. Phys. A 18 (2003) 19 [hep-th/0205143] [SPIRES].

[9] M.B. Halpern and C. Helfgott, Extended operator algebra and reducibility in the WZW permutation orbifolds, Int. J. Mod. Phys. A 18 (2003) 1773 [hep-th/0208087] [SPIRES].

[10] O. Ganor, M.B. Halpern, C. Helfgott and N.A. Obers, The outer-automorphic WZW orbifolds on $\mathrm{SO}(2 N)$, including five triality orbifolds on $\mathrm{SO}(8)$, JHEP 12 (2002) 019 [hep-th/0211003] [SPIRES].

[11] J. deBoer, M.B. Halpern and C. Helfgott, Twisted Einstein Tensors and Orbifold Geometry, Int. J. Mod. Phys. A 18 (2003) 3489 [hep-th/0212275] [SPIRES]. 
[12] M.B. Halpern and C. Helfgott, Twisted open strings from closed strings: The WZW orientation orbifolds, Int. J. Mod. Phys. A 19 (2004) 2233 [hep-th/0306014] [SPIRES].

[13] M.B. Halpern and C. Helfgott, On the target-space geometry of open-string orientationorbifold sectors, Annals Phys. 310 (2004) 302 [hep-th/0309101] [SPIRES].

[14] M.B. Halpern and C. Helfgott, A basic class of twisted open WZW strings, Int. J. Mod. Phys. A 19 (2004) 3481 [hep-th/0402108] [SPIRES].

[15] M.B. Halpern and C. Helfgott, The general twisted open WZW string, Int. J. Mod. Phys. A 20 (2005) 923 [hep-th/0406003] [SPIRES].

[16] M.B. Halpern, The orbifolds of permutation-type as physical string systems at multiples of $c=26$. I: Extended actions and new twisted world-sheet gravities, JHEP 06 (2007) 068 [hep-th/0703044] [SPIRES].

[17] M.B. Halpern, The Orbifolds of Permutation-Type as Physical String Systems at Multiples of $c=26$ II. The Twisted BRST Systems of $\hat{c}=52$ Matter, Int. J. Mod. Phys. A 22 (2007) 4587 [hep-th/0703208] [SPIRES].

[18] M.B. Halpern, The orbifolds of permutation-type as physical string systems at multiples of $c=26$. III: The spectra of $\hat{c}=52$ strings, Nucl. Phys. B 786 (2007) 297 [arXiv:0704.1540] [SPIRES].

[19] M.B. Halpern, The orbifolds of permutation-type as physical string systems at multiples of $c=26$ IV. orientation orbifolds include orientifolds, Phys. Rev. D 76 (2007) 026004 [arXiv: 0704.3667] [SPIRES].

[20] M.B. Halpern, The Orbifolds of Permutation-Type as Physical String Systems at Multiples of $c=26$ V. Cyclic Permutation Orbifolds, J. Math. Phys. 48 (2007) 122301 [arXiv:0705.2062] [SPIRES].

[21] P. Goddard and C.B. Thorn, Compatibility of the Dual Pomeron with Unitarity and the Absence of Ghosts in the Dual Resonance Model, Phys. Lett. B 40 (1972) 235 [SPIRES].

[22] I.V. Tyutin, Gauge Invariance of Field Theory and Statistical Physics in Operator Formalism, LEBEDEV-1975-39.

[23] C. Becchi, A. Rouet and R. Stora, Renormalization of Gauge Theories, Annals Phys. 98 (1976) 287 [SPIRES].

[24] K. Bardakci and M.B. Halpern, New dual quark models, Phys. Rev. D 3 (1971) 2493 [SPIRES].

[25] M.B. Green, J.H. Schwarz and E. Witten, Superstring Theory, Cambridge University Press (1987). 